\title{
Showcasing $H H$ production: Benchmarks for the LHC and HL-LHC
}

\author{
Philipp Basler, ${ }^{1, *}$ Sally Dawson, ${ }^{2, \dagger}$ Christoph Englert, ${ }^{3, \star}$ and Margarete Mühlleitner ${ }^{1, \S}$ \\ ${ }^{1}$ Institute for Theoretical Physics, Karlsruhe Institute of Technology, 76128 Karlsruhe, Germany \\ ${ }^{2}$ Department of Physics, Brookhaven National Laboratory, Upton, New York 11973, USA \\ ${ }^{3}$ SUPA, School of Physics and Astronomy, University of Glasgow, Glasgow G12 8QQ, United Kingdom
}

(Received 11 January 2019; published 29 March 2019)

\begin{abstract}
Current projections suggest that the LHC will have only limited sensitivity to di-Higgs production in the Standard Model (SM), possibly even after the completion of its high-luminosity phase. Multi-Higgs final states play a fundamental role in many extensions of the SM as they are intrinsically sensitive to modifications of the Higgs sector. Therefore, any new observation in multi-Higgs final states could be linked to a range of beyond the SM (BSM) phenomena that are not sufficiently addressed by the SM. Extensions of the Higgs sector typically lead to new phenomenological signatures in multi-Higgs final states that are vastly different from the SM expectation. In this work, we provide a range of signature-driven benchmark points for resonant and nonresonant BSM di-Higgs production that motivate non-SM kinematic correlations and multifermion discovery channels. Relying on theoretically well-motivated assumptions, special attention is devoted to the particular case where the presence of new physics will dominantly manifest itself in multi-Higgs final states.
\end{abstract}

DOI: 10.1103/PhysRevD.99.055048

\section{INTRODUCTION}

The Higgs precision spectroscopy program that ensued after the discovery of the Higgs boson in $2012[1,2]$ has assumed a central role in particle physics over the past years. One reason why measurements of the Higgs' couplings and its properties have become the focus of searches for physics beyond the Standard Model (BSM) is the lack of conclusive hints for new interactions in the plethora of BSM searches performed by the ATLAS and CMS experiments. The Higgs boson as the direct implication of electroweak symmetry breaking is typically considered as a harbinger of new physics due to its special role in the unitarization of scattering amplitudes at high energy [3-6] and its relation to the naturalness of the electroweak scale [7], to only name a couple of examples.

Although modifications of Higgs physics at the $\mathrm{TeV}$ scale of this size are still well within the limits set by recent $13 \mathrm{TeV}$ LHC measurements, now that the SM can be considered as complete no additional ultraviolet (UV) energy scale can be predicted from the SM alone. This will

\footnotetext{
"philipp.basler@kit.edu

dawson@bnl.gov

*christoph.englert@glasgow.ac.uk

§milada.muehlleitner@kit.edu
}

Published by the American Physical Society under the terms of the Creative Commons Attribution 4.0 International license. Further distribution of this work must maintain attribution to the author(s) and the published article's title, journal citation, and DOI. Funded by SCOAP ${ }^{3}$. become even more pressing if future coupling measurements are consistent with the SM expectation.

Additional requirements are more conveniently imposed in model-specific approaches, which try to mend apparent shortcomings of the SM such as the lack of a viable dark matter candidate or an insufficiently first-order electroweak phase transition to address the criteria of baryogenesis [8]. While model-independent approaches based on effective field theory (EFT) [9] can inform UV completions that address these questions through matching calculations, the appearance of novel phenomenological signatures such as resonances or thresholds within the LHC's kinematic coverage typically fall outside the region of reliability of these techniques.

A process that highlights the shortcomings of EFT methods in the presence of thresholds already in the SM context is di-Higgs production $p p \rightarrow h h$ (see e.g., Refs. [10,11] for recent summaries). Therefore, EFT methods for $g g h^{n}$ that can be summarized as [12-16]

$$
\begin{aligned}
\mathcal{L} & =\frac{\alpha_{s}}{12 \pi} G^{a \mu \nu} G_{\mu \nu}^{a} \log \left(1+\frac{h}{v}\right) \\
& =\frac{\alpha_{s}}{12 \pi} G^{a \mu \nu} G_{\mu \nu}^{a}\left(\frac{h}{v}-\frac{h^{2}}{v^{2}}\right)+\mathcal{O}\left(h^{3}\right)
\end{aligned}
$$

reflect the destructive interference between the top triangle and box diagrams of Fig. 1. While it can be assumed that the Higgs-top coupling can be accessed at the LHC in the range of $\sim 10 \%$ and the intermediate top mass scale is under theoretical control at the next-to-leading-order (NLO) 


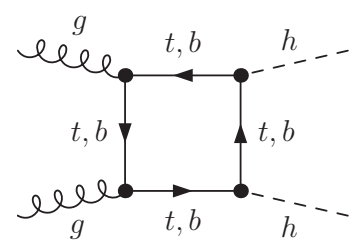

(a)

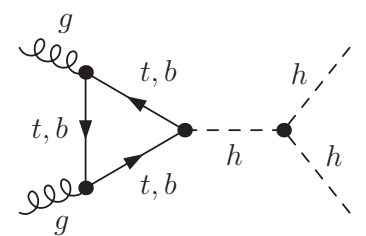

(b)
FIG. 1. Feynman diagrams contributing to $g g \rightarrow h h$ production. Although the bottom-quark contribution is included, it is entirely negligible.

level ${ }^{1}$ [17-23], the situation for the trilinear coupling is less clear as it probes a direction in the dimension-six linear EFT space $\sim c_{6}\left(H^{\dagger} H\right)^{3} / \Lambda^{2}$ (we denote the SM Higgs doublet with $H$ in the following) [24]. As $c_{6}$ is essentially a free parameter unless a matching calculation is performed, its size is only limited by technical considerations related to perturbation theory, on which we need to rely to make phenomenological predictions. This has raised the question of how large trilinear coupling modifications can be [25-28] to inform di-Higgs investigations. Current CMS projections show that a sensitivity of $\lesssim 0.5 \sigma$ at $3 \mathrm{ab}^{-1}$ [29] seems a realistic target at the LHC. Recent theoretical studies have been slightly more optimistic [30-32], but the Higgs trilinear coupling will only be understood in great detail at large luminosity, in particular in the light of possible top-quark Yukawa coupling modifications [33]. As a consequence, di-Higgs production is a main motivation for considering a major energy upgrade of the LHC or a future hadron collider [34-39].

In concrete UV scenarios that address fundamental BSM questions, typically a number of exotic states appear in the spectrum that will not only impact the "standard" single-Higgs phenomenology at the LHC but can also lead to even more dramatic changes in the production of multiple Higgs bosons. For instance, the triangle and box diagrams probe different aspects of top physics in extended top sectors [40-44].

Interleaving modifications of single-Higgs physics with theoretically well-motivated UV considerations can therefore turn di-Higgs production into a strong probe of new physics: new kinematic features can appear that motivate new final states and search strategies that are not currently considered, e.g., di-Higgs production can be enhanced or suppressed. Such phenomenological modifications become particularly relevant when extrapolations of standard singleHiggs channels do not show a significant departure from their SM expectation in these scenarios.

We will address these questions in this work using a particular set of models that allow us to contrast precise theoretical and phenomenological requirements with concrete predictions of single- and multi-Higgs production.

\footnotetext{
${ }^{1}$ A recent summary of higher-order corrections to Higgs pair production can be found in Ref. [17].
}

Imposing, e.g., a strong first-order electroweak phase transition, dark matter constraints, electric dipole measurements and consistency with current Higgs coupling measurements as well as an extrapolation thereof, we discuss the results of a comprehensive scan of the models' parameter space with a particular emphasis on the relevance of multi-Higgs final states. We distill this scan into a number of representative benchmark points of BSM theories that highlight the importance of di-Higgs measurements in the future. In passing, we discuss how non-SM signatures are correlated with modifications of singleHiggs physics BSM effects (or a lack of the latter). In turn, this also allows us to formulate an upper limit of the SM-like di-Higgs production cross section in these models when there are no conclusive hints for new physics in single Higgs phenomenology.

This work is structured as follows. In Sec. II, we outline the models that we consider for the purpose of this work. These are the $\mathrm{CP}$-violating two-Higgs-doublet model $(\mathrm{C} 2 \mathrm{HDM})$ and the next-to-minimal supersymmetric extension (NMSSM). Both models are special in the sense that they feature extended Higgs sectors that allow for Higgs-toHiggs decays, decays into final states with different Higgs bosons, or even cascade Higgs-to-Higgs decays (see also Ref. [45]). In Sec. III we outline the details of our scan over the model space and discuss the overall scan results. We present our benchmarks for the C2HDM and NMSSM together with their phenomenological properties in Sec. IV. We conclude in Sec. V.

\section{THE MODELS}

\section{A. The C2HDM}

The 2HDM [46-48] is obtained from the SM by adding a second $S U(2)_{L}$ Higgs doublet. The Higgs potential of a general $2 \mathrm{HDM}$ with a softly broken $\mathbb{Z}_{2}$ symmetry, under which $\Phi_{1} \rightarrow \Phi_{1}$ and $\Phi_{2} \rightarrow-\Phi_{2}$, can be written as

$$
\begin{aligned}
V= & m_{11}^{2}\left|\Phi_{1}\right|^{2}+m_{22}^{2}\left|\Phi_{2}\right|^{2} \\
& -\left(m_{12}^{2} \Phi_{1}^{\dagger} \Phi_{2}+\text { H.c. }\right)+\frac{\lambda_{1}}{2}\left(\Phi_{1}^{\dagger} \Phi_{1}\right)^{2} \\
& +\frac{\lambda_{2}}{2}\left(\Phi_{2}^{\dagger} \Phi_{2}\right)^{2}+\lambda_{3}\left(\Phi_{1}^{\dagger} \Phi_{1}\right)\left(\Phi_{2}^{\dagger} \Phi_{2}\right) \\
& +\lambda_{4}\left(\Phi_{1}^{\dagger} \Phi_{2}\right)\left(\Phi_{2}^{\dagger} \Phi_{1}\right)+\left[\frac{\lambda_{5}}{2}\left(\Phi_{1}^{\dagger} \Phi_{2}\right)^{2}+\text { H.c. }\right] .
\end{aligned}
$$

The absence of flavor-changing neutral currents is guaranteed by extending the $\mathbb{Z}_{2}$ symmetry to the fermions. Depending on the $\mathbb{Z}_{2}$ charge assignments, there are four phenomenologically different types of $2 \mathrm{HDMs}$ that are summarized in Table I. Hermiticity of the potential requires all parameters to be real, except for $\lambda_{5}$ and $m_{12}^{2}$. If they have different unrelated complex phases we are in the framework of the complex or $C P$-violating 2HDM [49], which 
TABLE I. The four Yukawa types of the $\mathbb{Z}_{2}$-symmetric $2 \mathrm{HDM}$ defined by the Higgs doublet that couples to each kind of fermions.

\begin{tabular}{lccc}
\hline \hline & $u$-type & $d$-type & Leptons \\
\hline Type I (T1) & $\Phi_{2}$ & $\Phi_{2}$ & $\Phi_{2}$ \\
Type II (T2) & $\Phi_{2}$ & $\Phi_{1}$ & $\Phi_{1}$ \\
Lepton-specific & $\Phi_{2}$ & $\Phi_{2}$ & $\Phi_{1}$ \\
Flipped & $\Phi_{2}$ & $\Phi_{1}$ & $\Phi_{2}$ \\
\hline \hline
\end{tabular}

depends on ten real parameters. In the description of the C2HDM we follow the conventions of Ref. [50]. The vacuum expectation values (VEVs) developed by the Higgs doublets after electroweak symmetry breaking could in principle be complex in the C2HDM. Since the phase can be removed by a basis change [49], we set it to zero without loss of generality. In terms of the complex charged fields $\phi_{i}^{+}$and the real neutral $C P$-even and $C P$-odd fields $\rho_{i}$ and $\eta_{i}(i=1,2)$, respectively, the Higgs doublets are given by

$$
\Phi_{1}=\left(\begin{array}{c}
\phi_{1}^{+} \\
\frac{v_{1}+\rho_{1}+i \eta_{1}}{\sqrt{2}}
\end{array}\right) \quad \text { and } \quad \Phi_{2}=\left(\begin{array}{c}
\phi_{2}^{+} \\
\frac{v_{2}+\rho_{2}+i \eta_{2}}{\sqrt{2}}
\end{array}\right) \text {, }
$$

where $v_{1}$ and $v_{2}$ denote the VEVs of the two Higgs doublets $\Phi_{1}$ and $\Phi_{2}$, respectively, and $v_{1}^{2}+v_{2}^{2}=v^{2}$ with the SM VEV $v \approx 246 \mathrm{GeV}$. The ratio of the VEVs is parametrized by the mixing angle $\beta$,

$$
\tan \beta \equiv t_{\beta}=\frac{v_{2}}{v_{1}} .
$$

The minimum conditions obtained from the requirement that the minimum of the potential is given by $\left\langle\Phi_{i}\right\rangle=\left(0, v_{i} / \sqrt{2}\right)^{T}$, can be used to trade the parameters $m_{11}^{2}$ and $m_{22}^{2}$ for $v_{1}$ and $v_{2}$. They also yield a relation between the imaginary parts of $m_{12}^{2}$ and $\lambda_{5}$, so that one of the ten parameters is fixed. The neutral Higgs mass eigenstates $H_{i}(i=1,2,3)$ are obtained from the neutral components of the C2HDM basis, $\rho_{1,2}$ and $\rho_{3} \equiv$ $(1 / \sqrt{2})\left(-\sin \beta \eta_{1}+\cos \beta \eta_{2}\right)$ via the rotation ${ }^{2}$

$$
\left(\begin{array}{l}
H_{1} \\
H_{2} \\
H_{3}
\end{array}\right)=R\left(\begin{array}{l}
\rho_{1} \\
\rho_{2} \\
\rho_{3}
\end{array}\right)
$$

The matrix $R$ diagonalizes the mass matrix $\mathcal{M}$ of the neutral states,

$$
R \mathcal{M}^{2} R^{T}=\operatorname{diag}\left(m_{H_{1}}^{2}, m_{H_{2}}^{2}, m_{H_{3}}^{2}\right),
$$

\footnotetext{
${ }^{2}$ Actually, the field $\rho_{3}$ is equal to the $C P$-odd component of the second Higgs doublet in the Higgs basis [51,52].
}

where $m_{H_{1}} \leq m_{H_{2}} \leq m_{H_{3}}$ denote the masses of the neutral Higgs bosons. Introducing the abbreviations $s_{i} \equiv \sin \alpha_{i}$ and $c_{i} \equiv \cos \alpha_{i}$ with

$$
-\frac{\pi}{2} \leq \alpha_{i}<\frac{\pi}{2}
$$

the mixing matrix $R$ can be parametrized as

$$
R=\left(\begin{array}{ccc}
c_{1} c_{2} & s_{1} c_{2} & s_{2} \\
-\left(c_{1} s_{2} s_{3}+s_{1} c_{3}\right) & c_{1} c_{3}-s_{1} s_{2} s_{3} & c_{2} s_{3} \\
-c_{1} s_{2} c_{3}+s_{1} s_{3} & -\left(c_{1} s_{3}+s_{1} s_{2} c_{3}\right) & c_{2} c_{3}
\end{array}\right) .
$$

In total, the $\mathrm{C} 2 \mathrm{HDM}$ has nine independent parameters that we choose to be [53]

$v, \quad t_{\beta}, \quad \alpha_{1,2,3}, \quad m_{H_{i}}, \quad m_{H_{j}}, \quad m_{H^{ \pm}}, \quad \operatorname{Re}\left(m_{12}^{2}\right)$.

The $m_{H_{i}}$ and $m_{H_{j}}$ denote any of the three neutral Higgs boson masses. The third mass is not independent and is calculated from the other parameters [53]. For further details, in particular also on the couplings of the C2HDM, see Ref. [54].

\section{B. The NMSSM}

Supersymmetric (SUSY) extensions require the introduction of at least a second Higgs doublet. In the NMSSM, the minimal field content with the doublet superfields $\hat{H}_{u}$ and $\hat{H}_{d}$ is extended by a complex superfield $\hat{S}$ (for reviews on the NMSSM, see for example Refs. [55,56]). The NMSSM Higgs potential is derived from the superpotential, the soft SUSY-breaking Lagrangian and the $D$-term contributions. The scale-invariant NMSSM superpotential reads in terms of the hatted superfields

$$
\begin{aligned}
\mathcal{W}= & \lambda \hat{S} \hat{H}_{u} \hat{H}_{d}+\frac{\kappa}{3} \hat{S}^{3}+h_{t} \hat{Q}_{3} \hat{H}_{u} \hat{t}_{R}^{c} \\
& -h_{b} \hat{Q}_{3} \hat{H}_{d} \hat{b}_{R}^{c}-h_{\tau} \hat{L}_{3} \hat{H}_{d} \hat{\tau}_{R}^{c} .
\end{aligned}
$$

For simplicity, we only included here the third-generation fermion superfields, given by the left-handed doublet quark $\left(\hat{Q}_{3}\right)$, and lepton $\left(\hat{L}_{3}\right)$ superfields as well as right-handed singlet quark $\left(\hat{t}_{R}^{c}, \hat{b}_{R}^{c}\right)$ and lepton $\left(\hat{\tau}_{R}^{c}\right)$ superfields. The soft SUSY-breaking Lagrangian

$$
\begin{aligned}
-\mathcal{L}_{\text {mass }}= & m_{H_{u}}^{2}\left|H_{u}\right|^{2}+m_{H_{d}}^{2}\left|H_{d}\right|^{2}+m_{S}^{2}|S|^{2}+m_{\tilde{Q}_{3}}^{2}\left|\tilde{Q}_{3}^{2}\right| \\
& +m_{\tilde{t}_{R}}^{2}\left|\tilde{t}_{R}^{2}\right|+m_{\tilde{b}_{R}}^{2}\left|\tilde{b}_{R}^{2}\right|+m_{\tilde{L}_{3}}^{2}\left|\tilde{L}_{3}^{2}\right|+m_{\tilde{\tau}_{R}}^{2}\left|\tilde{\tau}_{R}^{2}\right|,
\end{aligned}
$$

contains the mass terms $m_{x}$ for the Higgs $\left(x=H_{u}, H_{d}, S\right)$ and sfermion $\left(x=\tilde{Q}_{3}, \tilde{t}_{R}, \tilde{b}_{R}, \tilde{L}_{3}, \tilde{\tau}_{R}\right)$ fields, obtained from the complex scalar components of the superfields. The Lagrangian with the trilinear soft SUSY-breaking interactions $A_{\lambda, k, t, b, \tau}$ between the sfermion and Higgs fields reads 


$$
\begin{aligned}
-\mathcal{L}_{\text {tril }}= & \lambda A_{\lambda} H_{u} H_{d} S+\frac{1}{3} \kappa A_{\kappa} S^{3}+h_{t} A_{t} \tilde{Q}_{3} H_{u} \tilde{t}_{R}^{c} \\
& -h_{b} A_{b} \tilde{Q}_{3} H_{d} \tilde{b}_{R}^{c}-h_{\tau} A_{\tau} \tilde{L}_{3} H_{d} \tilde{\tau}_{R}^{c}+\text { H.c. }
\end{aligned}
$$

The contribution to soft SUSY breaking from the gaugino mass parameters $M_{1,2,3}$ of the bino $(\tilde{B})$, winos $(\tilde{W})$ and gluinos $(\tilde{G})$, respectively, is given by

$$
\begin{aligned}
-\mathcal{L}_{\text {gauginos }}= & \frac{1}{2}\left[M_{1} \tilde{B} \tilde{B}+M_{2} \sum_{a=1}^{3} \tilde{W}^{a} \tilde{W}_{a}\right. \\
& \left.+M_{3} \sum_{a=1}^{8} \tilde{G}^{a} \tilde{G}_{a}+\text { H.c. }\right] .
\end{aligned}
$$

Expanding the tree-level scalar potential around the nonvanishing VEVs of the Higgs doublet and singlet fields,

$$
\begin{aligned}
H_{d} & =\left(\begin{array}{c}
\left(v_{d}+h_{d}+i a_{d}\right) / \sqrt{2} \\
h_{d}^{-}
\end{array}\right), \\
H_{u} & =\left(\begin{array}{c}
h_{u}^{+} \\
\left(v_{u}+h_{u}+i a_{u}\right) / \sqrt{2}
\end{array}\right), \quad S=\frac{v_{s}+h_{s}+i a_{s}}{\sqrt{2}},
\end{aligned}
$$

leads to the Higgs mass matrices for the three scalar $\left(h_{d}, h_{u}\right.$, $\left.h_{s}\right)$, the three pseudoscalar $\left(a_{d}, a_{u}, a_{s}\right)$ and the charged Higgs states $\left(h_{u}^{ \pm}, h_{d}^{\mp}\right)$. We choose the VEVs $v_{u}, v_{d}$ and $v_{s}$ to be real and positive. The three $C P$-even mass eigenstates $H_{i}(i=1,2,3)$ are obtained from the interaction states through rotation with the orthogonal matrix $\mathcal{R}^{S}$ that diagonalizes the $3 \times 3$ mass matrix squared of the $C P$ even fields,

$$
\left(H_{1}, H_{2}, H_{3}\right)^{T}=\mathcal{R}^{S}\left(h_{d}, h_{u}, h_{s}\right)^{T} .
$$

The mass eigenstates are ordered by ascending mass, $M_{H_{1}} \leq M_{H_{2}} \leq M_{H_{3}}$. The $C P$-odd mass eigenstates $A_{1}$ and $A_{2}$ are obtained from a rotation $\mathcal{R}^{G}$ that separates the Goldstone boson, followed by a rotation $\mathcal{R}^{P}$ into the mass eigenstates,

$$
\left(A_{1}, A_{2}, G\right)^{T}=\mathcal{R}^{P} \mathcal{R}^{G}\left(a_{d}, a_{u}, a_{s}\right)^{T} .
$$

They are also ordered by ascending mass, $M_{A_{1}} \leq M_{A_{2}}$. Altogether, the NMSSM Higgs spectrum consists of seven physical Higgs states, three neutral $C P$-even, two neutral $C P$-odd and two charged Higgs bosons. We use the three minimization conditions of the scalar potential to replace the soft SUSY-breaking masses squared for $H_{u}, H_{d}$ and $S$ in $\mathcal{L}_{\text {mass }}$ by the remaining parameters of the tree-level potential so that the set of six parameters parametrizing the treelevel NMSSM Higgs sector is given by

$$
\lambda, \quad \kappa, \quad A_{\lambda}, \quad A_{\kappa}, \quad \tan \beta=v_{u} / v_{d}, \quad \mu_{\mathrm{eff}}=\lambda v_{s} / \sqrt{2} .
$$

The sign conventions are such that $\lambda$ and $\tan \beta$ are positive, while $\kappa, A_{\lambda}, A_{\kappa}$ and $\mu_{\text {eff }}$ can take both signs. Note that the Higgs boson masses are not input parameters, but dependent parameters calculated from the input values. The inclusion of higher-order corrections in the Higgs boson masses is crucial here to shift the mass of the SM-like Higgs boson to the observed value of $125 \mathrm{GeV}$.

\section{DETAILS OF THE SCAN}

\section{A. The C2HDM scan}

The benchmark points ${ }^{3}$ provided in this paper have to satisfy theoretical and experimental constraints. In order to find valid points, we perform a scan in the C2HDM parameter space and additionally require the mass of one of the Higgs bosons, to be identified with the SM-like one and denoted by $h$, to be $m_{h}=125.09 \mathrm{GeV}$ [59]. The scan ranges are summarized in Table II. For simplicity, we only consider the C2HDM type 1 (T1) and type 2 (T2), which cover to a large extent the phenomenological effects to be expected in the C2HDM. Since physical parameter points with $\operatorname{Re}\left(m_{12}^{2}\right)<0$ are extremely rare, though possible, we neglect them in our scan. We test for compatibility with the flavor constraints on $R_{b}[60,61]$ and $B \rightarrow X_{s} \gamma$ [61-65] as $2 \sigma$ exclusion bounds in the $m_{H^{ \pm}}-\tan \beta$ plane. In accordance with Ref. [65] we therefore require $m_{H^{ \pm}}$to be above $580 \mathrm{GeV}$ in the C2HDM T2, whereas in the C2HDM T1 this bound is much weaker and depends more strongly on $\tan \beta$. We verify agreement with the electroweak precision data by using the oblique parameters $S, T$ and $U$ (the 2HDM formulas are given in Refs. [48,66]) for which we demand $2 \sigma$ compatibility with the SM fit [67], including the full correlation among the three parameters. We require perturbative unitarity to hold at tree level. The third neutral Higgs boson mass $m_{H_{j} \neq H_{i}, h}$, which is not an independent input parameter in the $\mathrm{C} 2 \mathrm{HDM}$, but rather calculated from the other input values, is demanded to lie in the interval

$$
10 \mathrm{GeV} \leq m_{H_{j}}<1.5 \mathrm{TeV} .
$$

To avoid degenerate Higgs signals, we additionally impose $m_{H_{i} \neq h}$ to be $5 \mathrm{GeV}$ away from $125 \mathrm{GeV}$. For the SM input parameters we use $[68,69]$

$$
\begin{array}{rlrl}
\alpha\left(M_{Z}\right) & =1 / 127.92, \quad & \alpha_{s}^{\overline{\mathrm{MS}}}\left(M_{Z}\right)=0.118, \\
M_{Z} & =91.187 \mathrm{GeV}, \quad M_{W}=80.358 \mathrm{GeV}, \\
m_{t} & =172.5 \mathrm{GeV}, \quad m_{b}^{\overline{\mathrm{MS}}}\left(m_{b}^{\overline{\mathrm{MS}}}\right)=4.18 \mathrm{GeV}, \\
m_{\tau} & =1.777 \mathrm{GeV} .
\end{array}
$$

The remaining light quark and lepton masses have been set to $[68,69]$

${ }^{3}$ 2HDM benchmarks for double Higgs production can be found in Refs. [57,58]. 
TABLE II. Input parameters for the C2HDM scan, where all parameters are varied independently between the given minimum and maximum values. The two minimum values of the charged Higgs mass range refer to the scan in the C2HDM T1 and T2, respectively. For more details, see text.

\begin{tabular}{cccccc}
\hline \hline & $t_{\beta}$ & $\alpha_{1,2,3}$ & $\operatorname{Re}\left(m_{12}^{2}\right)\left[\mathrm{TeV}^{2}\right]$ & $m_{H^{ \pm}}[\mathrm{TeV}]$ & $m_{H_{i} \neq h}[\mathrm{TeV}]$ \\
\hline $\operatorname{Min}$ & 0.8 & $-\frac{\pi}{2}$ & 0 & $0.15 / 0.59$ & 0.01 \\
$\operatorname{Max}$ & 20 & $\frac{\pi}{2}$ & 0.5 & 1.5 & 1.5 \\
\hline \hline
\end{tabular}

$m_{e}=0.510998928 \mathrm{MeV}, \quad m_{\mu}=105.6583715 \mathrm{MeV}$,

$m_{u}=100 \mathrm{MeV}, \quad m_{d}=100 \mathrm{MeV}$,

$m_{s}=100 \mathrm{MeV}$.

In order to perform the scans and find valid parameter points we use the program Scanners [70,71]. Besides the above-mentioned constraints it also tests for the potential to be bounded from below and uses the tree-level discriminant of Ref. [72] to enforce the electroweak vacuum to be the global minimum of the tree-level Higgs potential. Agreement with the Higgs exclusion limits from LEP, Tevatron and LHC is checked by using HiggsBounds5 .2.0 [73-75] and with the Higgs rates by using HiggsSignals2 .2.1 [76]. The required decay widths and branching ratios are obtained from the C2HDM implementation C2HDM_HDECAY [54] in HDECAY [77,78]. In the production cross sections we include the QCD corrections taken over from the SM and the MSSM, where available. Electroweak corrections are consistently neglected both in production and decays. The size of the electroweak corrections is typically of the order of 10-20\%. They can, however, also be considerably larger in the case of parametrically enhanced corrections due to large involved couplings and/or in the case of light particles in the loop (see e.g., the results for the EW corrections to Higgs-toHiggs decays in the 2HDM of Ref. [79]). For more details on the production cross sections, see Refs. [54,80].

Working in the C2HDM, we also make sure to be in agreement with the measurements of the electric dipole moment $(\mathrm{EDM})$, where the strongest constraint originates from the electron EDM [81]. We take the experimental limit given by the ACME Collaboration [82]. Finally, we also investigate for the $\mathrm{C} 2 \mathrm{HDM}$ if the parameters of the final data set induce a strong first-order phase transition, a necessary condition for successful baryogenesis [8,83,84], by using the $\mathrm{C}++$ code BSMPT [85].

\section{B. The NMSSM scan}

In order to find benchmark points ${ }^{4}$ that are compatible with the recent experimental constraints we proceed as

\footnotetext{
${ }^{4}$ For NMSSM benchmarks for double Higgs production from Higgs-to-Higgs decays, see Ref. [86].
}

described in Refs. [86-88], where further details can also be found. We perform a scan in the NMSSM parameter space for the scan ranges summarized in Table III. The remaining mass parameters of the third-generation sfermions not listed in the table are chosen as

$m_{\tilde{t}_{R}}=m_{\tilde{Q}_{3}}, \quad m_{\tilde{\tau}_{R}}=m_{\tilde{L}_{3}} \quad$ and $\quad m_{\tilde{b}_{R}}=3 \mathrm{TeV}$.

The mass parameters of the first- and second-generation sfermions are set to

$m_{\tilde{u}_{R}, \tilde{c}_{R}}=m_{\tilde{d}_{R}, \tilde{s}_{R}}=m_{\tilde{Q}_{1,2}}=m_{\tilde{L}_{1,2}}=m_{\tilde{e}_{R}, \tilde{\mu}_{R}}=3 \mathrm{TeV}$.

The soft SUSY-breaking trilinear couplings of the first two generations are set equal to the corresponding values of the third generation. In order to ensure perturbativity we apply the rough constraint

$$
\lambda^{2}+\kappa^{2}<0.7^{2} .
$$

In accordance with the SUSY Les Houches Accord format $[89,90]$ the soft SUSY-breaking masses and trilinear couplings are understood as $\overline{\mathrm{DR}}$ parameters at the scale

$$
\mu_{R}=M_{s}=\sqrt{m_{\tilde{Q}_{3}} m_{\tilde{t}_{R}}} .
$$

The SM input parameters have been chosen as in the C2HDM scan, with the exception of the top-quark mass which has been set to $m_{t}=173.5 \mathrm{GeV}$. The small difference of $1 \mathrm{GeV}$ has no effect on the scan results.

The spectrum of the Higgs and SUSY particles including higher-order corrections is calculated with NMSSMTOols5 .2 . 0 [91-96] which also checks for the constraints from flavor and low-energy observables. It provides the input for HiggsBounds5 .2 . 0 [73-75] to check for compatibility with the exclusion bounds from the Higgs searches. The mass of one of the neutral $C P$-even Higgs bosons, identified with the SM-like Higgs boson denoted by $h$, has to lie in the range

$$
124 \mathrm{GeV} \leq m_{h} \leq 126 \mathrm{GeV},
$$

and the masses of all other Higgs bosons are demanded to be separated by at least $1 \mathrm{GeV}$ in order to avoid two overlapping signals. The signal strengths of this Higgs boson have to be in agreement with the signal strength fit of Ref. [97]. To sample a broader range of potentially viable parameter points, however, we inflate the $1 \sigma$ bands by a factor of 2. For the computation of the signal strengths we need the production cross section and branching ratios for the NMSSM Higgs bosons. To compute production through gluon fusion and $b \bar{b}$ annihilation, we take the SM cross sections and multiply them with the effective couplings obtained from NMSSMTOols. The SM values are calculated with SusHi $[98,99]$ and include in gluon fusion the NLO corrections with the full top-quark mass 
TABLE III. Input parameters for the NMSSM scan, where all parameters are varied independently between the given minimum and maximum values.

\begin{tabular}{llllllllllllllr}
\hline \hline & \multirow{2}{*}{$t_{\beta}$} & $\lambda$ & $\kappa$ & $M_{1}$ & $M_{2}$ & $M_{3}$ & $A_{t}$ & $A_{b}$ & $\begin{array}{c}A_{\tau} \\
\text { in } \mathrm{TeV}\end{array}$ & $m_{\tilde{Q}_{3}}$ & $m_{\tilde{L}_{3}}$ & $A_{\lambda}$ & $A_{\kappa}$ & $\mu_{\text {eff }}$ \\
\hline $\operatorname{Min}$ & 1 & 0 & -0.7 & 0.1 & 0.2 & 1.3 & -6 & -6 & -3 & 0.6 & 0.6 & -2 & -2 & -5 \\
$\operatorname{Max}$ & 50 & 0.7 & 0.7 & 1 & 2 & 7 & 6 & 6 & 3 & 4 & 4 & 2 & 2 \\
\hline \hline
\end{tabular}

dependence [100] as well as the next-to-next-to-leadingorder corrections in the heavy quark effective theory [101-105]. The next-to-next-to-next-to-leading-order corrections are taken into account in a threshold expansion [106-109] for Higgs masses below $300 \mathrm{GeV}$. For masses above $50 \mathrm{GeV}, b \bar{b}$ annihilation cross sections that match between the five- and four-flavor scheme are used, obtained in the soft-collinear effective theory [110,111]. They are in accordance with the results from Refs. [112,113]. For masses below $50 \mathrm{GeV}$, cross sections obtained in the Santander matching [114] are used, with the five-flavor scheme cross sections from Ref. [115] and the four-flavor scheme ones from Refs. [116-118]. The branching ratios are taken from NMSSMTOols and cross-checked against NMSSMCALC [119].

The parameter points also have to satisfy the bounds from SUSY searches at the LHC. The gluino mass and the lightest squark mass of the second generation are demanded to lie above $1.85 \mathrm{TeV}$, respectively (see Ref. [120]). The top squark and sbottom masses are required to be above $800 \mathrm{GeV}$, respectively, [120,121], the slepton masses above $400 \mathrm{GeV}$ [120] and the absolute value of the lightest chargino mass above $300 \mathrm{GeV}$ [122].

Through an interface with micrOMEGAS [96] we obtain the relic density which must not exceed the value measured by the Planck Collaboration [123]. The spin-independent nucleon-dark matter direct detection cross section, also provided by micrOMEGAS, is required not to violate the upper bound from the LUX experiment [124]. We furthermore test for compatibility with the direct detection limits from XENON1T [125] and check the dark matter annihilation cross section against the results provided by Fermi-LAT [126].

\section{Extrapolations}

We include a range of extrapolations of single-Higgs measurements in our discussion to identify an approximate "exclusion luminosity" (see below) at which single-Higgs measurements will start to become sensitive to a particular scenario and spectrum. This notion will allow us to put multi-Higgs final states in direct comparison with singleHiggs measurement expectations and identify interesting regions of parameter space.

In particular, we include projections for the $m_{h} \simeq$ $125 \mathrm{GeV}$ standard single-Higgs production modes, $g g \rightarrow h$ (gluon fusion), $q q \rightarrow h j j$ (weak boson fusion), $q q \rightarrow V h, V=W^{ \pm}, Z$ (Higgs radiation), $p p \rightarrow t \bar{t} h$ (associated production with top quarks), and consider decays $h \rightarrow Z Z, h \rightarrow W W, h \rightarrow \gamma \gamma, h \rightarrow b \bar{b}$ and $h \rightarrow \tau^{+} \tau^{-}$. For the decays $h \rightarrow \gamma \gamma, Z Z$ we use the CMS projections provided in Ref. [29]; these include the production modes gluon fusion, weak boson fusion and $t \bar{t} h$. We interpolate between different luminosities using a $\sqrt{\mathcal{L}}$ luminosity dependence at all times.

Projections for $h \rightarrow W W$ are obtained using Ref. [127] and we rescale these results taking into account the cross section differences between 13 and $8 \mathrm{TeV}$ using the results provided by the Higgs Cross Section Working Group [128]. For $h \rightarrow b \bar{b}$ we consider extrapolations based on $V h$ production [129], tith production [130] as well as weak boson fusion [131]. $h \rightarrow \tau \tau$ is based on Ref. [132], which agrees with the ECFA results of Ref. [29] upon projecting to $3 \mathrm{ab}^{-1}$.

The improved determination of the SM-like Higgs boson with $m_{h} \simeq 125 \mathrm{GeV}$ needs to be contrasted with additional coverage of Higgs-like searches for masses $m_{h} \neq 125 \mathrm{GeV}$. We include projections of existing resonance searches in $\gamma \gamma$ [133], $\tau \tau$ [134], $W W$ [135] and $Z Z$ [136] final states. By far the most constraining exotic searches result from $t \bar{t}$ resonance searches, and we extrapolate the results of Ref. [137]. This analysis is performed in the context of a $Z^{\prime}$ model and can therefore be interpreted as only a rough estimate of the search potential of $t \bar{t}$ Higgs resonances. To our knowledge no comprehensive analysis of exotic heavy Higgs masses is publicly available. This is mostly due to the dedicated interference between the background and the Higgs signal that also depends on the $C P$ character of the produced state [138]. This has a significant impact on the sensitivity of $t \bar{t}$ final states. Including such effects is beyond the scope of this work.

\section{Results}

Both in the C2HDM and the NMSSM the enlarged Higgs sector leads to a plethora of di-Higgs production processes. In particular, they feature processes with two different Higgs bosons in the final state. Compared to the $\mathrm{SM}$, the cross sections can be largely enhanced in the case of resonant production of a heavy Higgs boson that subsequently decays into a pair of lighter Higgs bosons, provided the Higgs self-coupling is not too small. Moreover, the different Higgs self-couplings themselves can enhance the cross section in view of the well-known 
fact that in the SM the triangle and box diagrams interfere destructively. Additionally, loops with bottom quarks may play a role in scenarios with enhanced down-type Yukawa couplings for large $\tan \beta$ in the NMSSM or the C2HDM T2. In the NMSSM loops with top-squark and sbottom quarks also contribute to Higgs pair production, and we furthermore have the possibility to produce a di-Higgs final state with pseudoscalars. These processes can yield even larger rates as has been discussed in detail in Ref. [87]. However, due to supersymmetry, the Higgs self-couplings are given in terms of the gauge couplings which limits deviations from the SM to some extent. This is not the case for the C2HDM, and thus effects different from those in the NMSSM may be expected here. In the NMSSM, on the other hand, it is possible for the final-state Higgs bosons to decay into non-SM final states like e.g., neutralinos, inducing signatures with phenomenologically interesting features. Altogether, both models provide a large playground for possible BSM effects in Higgs pair production that can be rather different.

Furthermore, we restrict ourselves to SM final states. Most of our results show the leading-order (LO) Higgs pair production cross sections. For the benchmark points we also computed the NLO QCD corrections in the limit of heavy loop particles. They typically increase the cross section by about a factor of 2 . We have implemented the NLO QCD corrections both for the NMSSM $^{5}$ [87] and C2HDM [140] in the FORTRAN code HPAIR ${ }^{6}$ that was originally designed to compute the SM and MSSM Higgs pair production at NLO QCD. All Higgs pair production processes have been computed at a c.m. energy of $\sqrt{s}=14 \mathrm{TeV}$, and we have adopted the CT14 parton densities [141] for the LO and NLO cross sections with $\alpha_{s}\left(M_{Z}\right)=0.118$ at LO and NLO. The renormalization scale has been set equal to $M_{H H} / 2$, where $M_{H H}$ generically denotes the invariant mass of the final-state Higgs pair. Consistent with the application of the heavy top-quark limit in the NLO QCD corrections, we neglect the bottom-quark loops in the LO cross section.

In view of the possibility of (largely) enhanced production of a pair of SM-like Higgs bosons, in the selection of valid scenarios, we also took into account limits set by LHC $4 b$ [142-144], $(2 b)(2 \tau)$ [145-147] and $(2 b)(2 \gamma)$ [148] final states from the production of a heavy scalar resonance that decays into two $125 \mathrm{GeV}$ Higgs bosons.

\section{1. $C 2 H D M$}

We start by discussing the possible sizes of Higgs pair production that are compatible with all present

\footnotetext{
${ }^{5}$ They only include the corrections to the top-quark loops. For NLO QCD corrections including also the squarks in the limit of vanishing external momenta, see Ref. [139].

${ }^{6}$ See M. Spira's website at http://tiger.web. psi.ch/proglist .html.
}

TABLE IV. Maximum cross section values in fb for LO gluon fusion into Higgs pairs, $\sigma\left(g g \rightarrow H_{i} H_{j}\right)$, in the C2HDM T1 and $\mathrm{T} 2$, with an exclusion luminosity $\geq 64 \mathrm{fb}^{-1}$ that satisfy all theoretical and experimental constraints described above.

\begin{tabular}{lcc}
\hline \hline$H_{i} H_{j} /$ model & $\mathrm{T} 1$ & $\mathrm{~T} 2$ \\
\hline$h h$ & 794 & 34.2 \\
$h H_{\downarrow}$ & 49.17 & 11.38 \\
$h H_{\uparrow}$ & 17.65 & 10.84 \\
$H_{\downarrow} H_{\downarrow}$ & 3196 & 0.18 \\
$H_{\downarrow} H_{\uparrow}$ & 12.58 & 0.11 \\
$H_{\uparrow} H_{\uparrow}$ & 7.10 & 0.18 \\
\hline \hline
\end{tabular}

experimental constraints. Table IV summarizes the maximum cross section values found in the sample of valid parameter points where we additionally applied the extrapolations of Sec. III C. Taking these into account, we only kept the points that are not excluded at $64 \mathrm{fb}^{-1}$, which corresponds approximately to the present luminosity acquired by the LHC experiments. We will come back to the role of the extrapolations below. In the following, we denote the SM-like Higgs boson with a mass of $125 \mathrm{GeV}$ as $h$, the lighter of the non-SM like neutral Higgs bosons is called $H_{\downarrow}$, and the heavier one is $H_{\uparrow}$. All cross sections are calculated at LO QCD and hence still increase by approximately a factor of 2 when QCD corrections are included.

The SM Higgs pair production cross section in gluon fusion amounts to $19.72 \mathrm{fb}$ at LO and $38.19 \mathrm{fb}^{\mathrm{L}}$ at $\mathrm{NLO}^{7}$ with a $K$ factor of $K=1.95$. Table IV shows that in both the $\mathrm{T} 1$ and $\mathrm{T} 2$ scenarios the maximum attained cross section for $h h$ production can exceed the SM value, in $\mathrm{T} 1$ by a factor of about 40 and in $\mathrm{T} 2$ by a factor of about 1.7. This is also the case at NLO, with NLO cross sections and $K$ factors for $h h$ production in the T1 and T2 models given by

$$
\begin{aligned}
& \mathrm{T} 1: \sigma(h h)^{\mathrm{NLO}}=1.64 \mathrm{pb}, \quad K=2.06, \\
& \mathrm{~T} 2: \sigma(h h)^{\mathrm{NLO}}=64.96 \mathrm{fb}, \quad K=1.94 .
\end{aligned}
$$

The reason for the large enhancement in $\mathrm{T} 1$ is the resonant production of the heavier Higgs bosons $H_{\downarrow}$ and $H_{\uparrow}$ with masses of 285 and $287 \mathrm{GeV}$, respectively, that subsequently decay into a pair of SM-like Higgs bosons. This is also the reason for the enhancement in $\mathrm{T} 2$, where the masses of the non-SM-like Higgs bosons amount to 794 and $798 \mathrm{GeV}$. The reason for the much smaller enhancement in $h h$ production in $\mathrm{T} 2$ compared to $\mathrm{T} 1$ is the overall heavier Higgs spectrum in T2. In particular, the intermediate heavy resonances in the $\mathrm{T} 2$ scenarios that can produce $h h$ in their decay usually fall into the heavy mass range where the

\footnotetext{
${ }^{7}$ This value differs from the one given in Ref. [18]. This is because we do not include top-quark mass effects here and use a different parton distribution function set.
} 

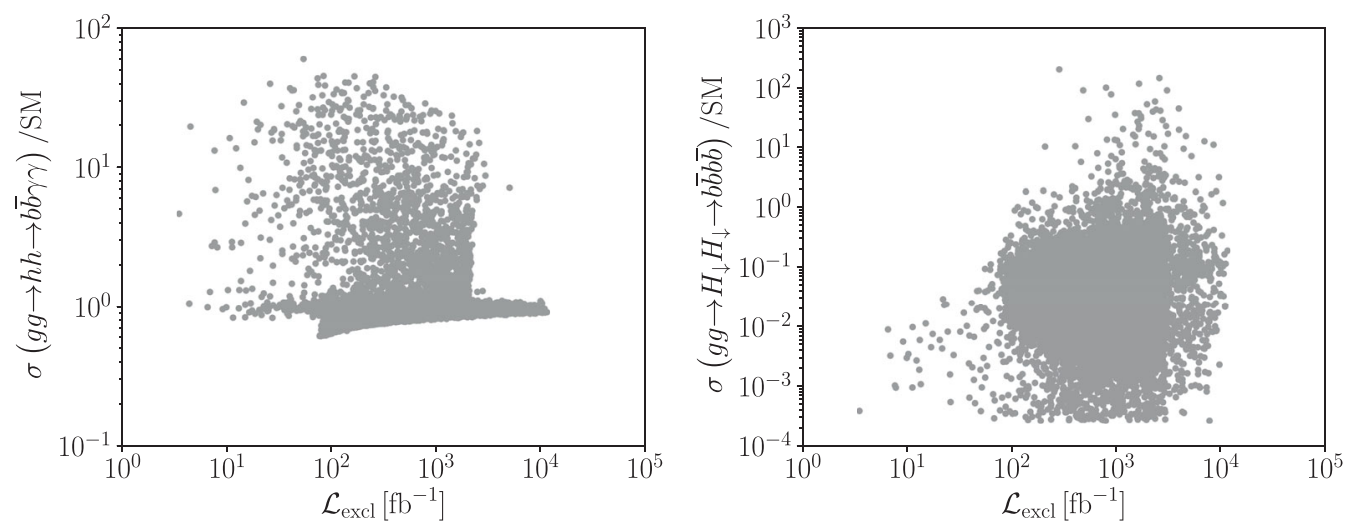

FIG. 2. C2HDM T1: Scatter plots for scenarios passing our applied constraints: Higgs pair production cross sections normalized to the SM value for SM-like Higgs pairs decaying into $(b \bar{b})(\gamma \gamma)$ (left) and light-non-SM-like Higgs pairs decaying into $(b \bar{b})(b \bar{b})($ right $)$ as a function of the exclusion luminosity.

ATLAS and CMS limits on the upper cross section for $(4 b)$ production drop rapidly, cf. [142,143]. In T1, furthermore, the maximum values of di-Higgs production processes involving $H_{\downarrow}$ can compete with SM Higgs pair production or even largely exceed it. Thus the production of an SMlike Higgs boson and $H_{\downarrow}$ can be larger by a factor of 2.5 . This final state is interesting as it is clearly a non-SM-like signature where the experiments can use the SM-like Higgs boson to calibrate or "tag" this exotic configuration. This does not apply for T2, however, where due to the experimental constraints, the non-SM-like Higgs bosons are in general heavier than in T1, inducing small di-Higgs production processes due to a much smaller phase space.

\section{Experimental accessibility and exclusion luminosity}

In order to assess the experimental accessibility of these cross sections, however, we need to look at their decay products. We therefore applied the narrow-width approximation and multiplied the produced Higgs bosons with their branching ratios in various SM final states. The most promising final states for the investigation of Higgs pair production at the LHC are the $(b \bar{b})(\gamma \gamma)$ [149], $(b \bar{b})(\tau \tau)$ [150-152] and $(b \bar{b})(b \bar{b})[150,153,154]$ final states (for other final states see also Refs. [155-157]). In Fig. 2 we show for all parameter points that pass our applied constraints for the C2HDM T1, the cross section values of SM Higgs pair production in the $(b \bar{b})(\gamma \gamma)$ final state (left) and for $H_{\downarrow} H_{\downarrow}$ production in the $(b \bar{b})(b \bar{b})$ final state (right) normalized to the corresponding SM values as a function of the exclusion luminosity. By the latter we define the luminosity at which this process would be excluded experimentally, based on the extrapolations described in Sec. III C. First of all we notice that the figures contain parameter points at lower luminosity that should have been excluded by HiggsBounds. The reason why they are there is that HiggsBounds relies on the published experimental results and cannot check for certain signatures that become relevant in BSM Higgs sectors. Thus there exist Higgs spectra with heavy Higgs bosons that dominantly decay into top-quark pairs. These would induce exotic four-top final states in heavy Higgs pair production. Such signatures compete, however, with single heavy Higgs production and subsequent decay into a top-quark pair. Applying our rough estimate on the exclusion power of the experiments for this process, based on the $Z^{\prime}$ data, such scenarios are excluded already, although they have been let through by HiggsBounds due to the lack of a dedicated experimental analysis for this. This shows the importance of experimental analyses investigating top pair final states from heavy Higgs production in order to properly assess the exclusion limits for BSM Higgs sectors, with dramatic effects on possible Higgs pair production signatures. While our rough extrapolation excludes about $0.6 \%$ of the $\mathrm{T} 1$ points for a luminosity of about $36 \mathrm{fb}^{-1}$, the effect is much larger for the T2 sample allowed by HiggsBounds. ${ }^{8}$ Here about $22 \%$ of the points would be excluded. This is because of the overall heavy non-SMlike Higgs bosons in T2 and their prominent decays into top-quark pairs.

As can be inferred from the figures in the C2HDM T1, the production of a SM-like Higgs pair with subsequent decay into $(b \bar{b})(\gamma \gamma)$ can exceed the SM rates by up to a factor of 60. This maximum enhancement factor is the same for all final states, as the branching ratios of the SM-like Higgs boson $h$ are almost the same as in the SM. In the following, we will use the quantity

$$
\Sigma_{X}=\sum_{i \in \mathrm{SM} \backslash\{h\}} \operatorname{BR}(X \rightarrow i),
$$

to classify whether a Higgs boson $X$ has a sizable non-SM branching ratio and decay phenomenology. If $\Sigma_{X} \simeq 1$ then the exotic states can be dominantly discovered in "standard" SM-Higgs-like decay channels, e.g., $X \rightarrow b \bar{b}$ or $t \bar{t}$ if the mass of $X$ permits such a decay.

\footnotetext{
${ }^{8}$ HiggsBounds takes into account data at $36 \mathrm{fb}^{-1}$.
} 
TABLE V. NMSSM: Maximum cross section values in fb for LO gluon fusion into Higgs pairs, $\sigma\left(g g \rightarrow H_{i} H_{j}\right)$ with an exclusion luminosity $\geq 64 \mathrm{fb}^{-1}$ that satisfy all theoretical and experimental constraints described above.

\begin{tabular}{lc}
\hline \hline$H_{i} H_{j}$ & NMSSM \\
\hline$h h$ & 67 \\
$h H_{\downarrow}$ & 26 \\
$h A_{\downarrow}$ & 493 \\
$h H_{\uparrow}$ & 25 \\
$H_{\downarrow} H_{\downarrow}$ & 4114 \\
$H_{\downarrow} H_{\uparrow}$ & 1.20 \\
$H_{\uparrow} H_{\uparrow}$ & 0.09 \\
$A_{\downarrow} A_{\downarrow}$ & 15894 \\
\hline \hline
\end{tabular}

In the $H_{\downarrow} H_{\downarrow}$ final state with both $H_{\downarrow}$ 's decaying into bottom quarks the enhancement can even be up to a factor of about 200. The point with the maximum enhancement corresponds to the one quoted in Table IV and the enhancement is due to the large di-Higgs production process of $3.2 \mathrm{pb}$ and a slightly enhanced branching ratio into $b$ quarks as compared to the SM. The same factor is found for the $(b \bar{b})(\tau \bar{\tau})$ final state. Due to a smaller branching ratio into photons, however, the maximum allowed enhancement in the $(b \bar{b})(\gamma \gamma)$ final state only amounts to a factor of up to 40 . The $H_{\downarrow}$ in this scenario has a mass of $m_{H_{\downarrow}}=131 \mathrm{GeV}$, and the mass of $H_{\uparrow}$ is $m_{H_{\uparrow}}=313 \mathrm{GeV}$. Its main branching ratios are $\operatorname{BR}\left(H_{\uparrow} \rightarrow Z H_{\downarrow}\right)=0.53$ and $\operatorname{BR}\left(H_{\uparrow} \rightarrow\right.$ $\left.H_{\downarrow} H_{\downarrow}\right)=0.46$. The maximum branching ratios of the charged Higgs boson with a mass of $m_{H^{+}}=312 \mathrm{GeV}$ are $\operatorname{BR}\left(H^{+} \rightarrow W^{+} H_{\downarrow}\right)=0.65$ and $\operatorname{BR}\left(H^{+} \rightarrow t \bar{b}\right)=0.34$. With its large di-Higgs production cross section and the large non-SM-like branching ratios, this parameter point is an interesting scenario for studying new physics effects (also beyond the Higgs pair events that we consider here).

All remaining di-Higgs production processes are less promising. Thus the enhancement factor for $h H_{\downarrow}$ production remains below 3 in the $4 b$ and $2 b 2 \tau$ final state and below 2 in the $2 b 2 \gamma$ final state. All other final states range below the SM values.

As can already be inferred from the maximum di-Higgs production values in T2, given in Table IV the situation looks much less promising in the C2HDM T2. There are very few points in $h h$ production with subsequent decays into the $(2 b)(2 \tau)$ and $4 b$ final states that exceed the SM rate, and only by a factor of about 2.4. The maximum enhancement found in the $(2 b)(2 \gamma)$ final state is about 2.4. All other final states lead to smaller rates than in the SM.

From these considerations we can conclude that there are promising di-Higgs signatures with large rates in the C2HDM T1 both for SM-like Higgs pair production but also for final states with non-SM-like Higgs bosons. The exotic Higgs bosons appear in SM-like final states, however, with different kinematic correlations due to different masses. This highlights the need to conduct Higgs pair analyses in a broad range of kinematic possibilities. Furthermore, the strict constraints on T2 scenarios, would exclude the model if di-Higgs signatures much larger than those in the SM are found.

\section{NMSSM}

In Table V we summarize for the NMSSM the maximum di-Higgs production cross section values found in the sample of valid parameter points that are not excluded at a luminosity of $64 \mathrm{fb}^{-1}$. All cross sections are calculated at LO QCD and hence still increase by approximately a factor of 2 when QCD corrections are included. By $A_{\downarrow}$ we denote the lighter of the two pseudoscalar Higgs bosons.

The reason for the large enhancement of $\sigma(g g \rightarrow h h)$ is the intermediate resonant production of heavy Higgs bosons $H_{\downarrow}$ and $H_{\uparrow}$ with subsequent decay into an SMlike Higgs pair. The $H_{\downarrow} H_{\downarrow}$ production cross section is so large because of the smallness of the $H_{\downarrow}$ mass, $m_{H_{\downarrow}}=$ $39.52 \mathrm{GeV}$. The enhancement in $h A_{\downarrow}$ production is due to

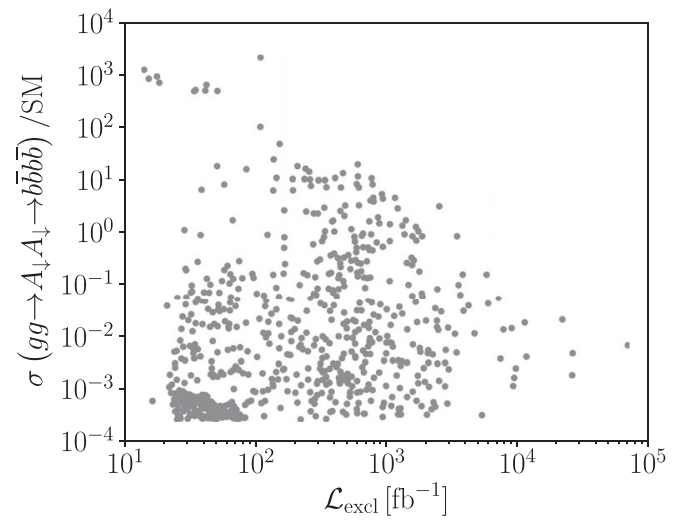

FIG. 3. NMSSM: Scatter plots for scenarios passing our applied constraints: Higgs pair production cross sections normalized to the SM value for SM-like Higgs pairs decaying into $(b \bar{b})(b \bar{b})$ (left) and $A_{\downarrow} A_{\downarrow}$ Higgs pairs decaying into $(b \bar{b})(b \bar{b})$ (right) as a function of the exclusion luminosity. 
the resonant $A_{2} \equiv A_{\uparrow}$ production with subsequent decay into $h A_{\downarrow}$. The huge enhancement in $A_{\downarrow} A_{\downarrow}$ production is on the one hand due to the smallness of the $A_{\downarrow}$ mass of $m_{A_{\downarrow}}=37 \mathrm{GeV}$, and on the other hand due to the resonant $H_{\uparrow}$ production with subsequent decay into $A_{\downarrow} A_{\downarrow}$ (the resonant $H_{\downarrow}$ production plays a minor role). Searches for relatively low-mass states are performed in the $\gamma \gamma$ [158] and $\tau \tau$ [159] channels, however, with rather limited sensitivity.

\section{Experimental accessibility and exclusion luminosity}

In Fig. 3 we show for all of the parameter points that pass our applied constraints, the NMSSM cross section values of SM Higgs pair production in the $(b \bar{b})(b \bar{b})$ final state (left) and for $A_{\downarrow} A_{\downarrow}$ production in the $(b \bar{b})(b \bar{b})$ final state (right) normalized to the corresponding SM values as a function of the exclusion luminosity.

As can be inferred from Fig. 3 (left), the $4 b$ final-state rates from SM-like Higgs pair production exceed the SM reference value by less than a factor of 10 and only for lower exclusion luminosities. Large enhancement factors are basically limited by the LHC upper limits on heavy resonant scalar production with subsequent decay into an SM-like Higgs pair. The situation looks even less promising in the production of an SM-like Higgs boson together with the lighter of the $C P$-even non-SM-like Higgs bosons, where only an enhancement factor slightly above 2.3 at most is found. This is the case for high exclusion luminosities beyond $1 \mathrm{ab}^{-1}$ so that nevertheless this process might be accessible at high luminosities. The situation is different in the production of $h A_{\downarrow}$. Because the lighter pseudoscalar can be relatively light and decays dominantly into $(b \bar{b})^{9}$ we can have enhancement factors above 10 up to about 45 in the $4 b$ state. This makes it particularly interesting, moreover in view of the exotic final state with two different Higgs masses in di-Higgs production. The enhancement factors can become huge in $A_{\downarrow} A_{\downarrow}$ production, which is mainly due to the lightness of $A_{\downarrow}$. In $4 b$ production it can be up to 1000 . For larger exclusion luminosities the enhancement factor can still be a factor of up to 10 , cf. Fig. 3 (right). In the $(b \bar{b})(\gamma \gamma)$ final state the enhancement can be larger than 100 up to about 360 .

Figure 4 nicely summarizes the situation of the enhanced di-Higgs cross sections involving very light Higgs bosons. It shows the production of $A_{\downarrow} A_{\downarrow}$ with subsequent decay in the $4 b$ final state normalized to the value of the corresponding process for the SM-like di-Higgs production, as a function of the mass of the light pseudoscalar. The color code denotes the exclusion luminosity. For very light masses below $125 \mathrm{GeV}$ (note that the gap around $125 \mathrm{GeV}$

\footnotetext{
${ }^{9}$ Note that typical trigger criteria are too selective to directly observe $p p \rightarrow A_{\downarrow} \rightarrow b \vec{b}$.
}

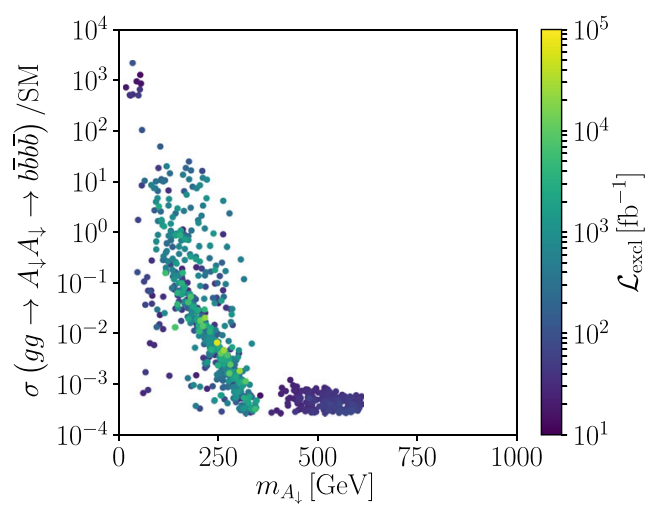

FIG. 4. NMSSM: Scatter plots for $4 b$ final-state rates from $A_{\downarrow} A_{\downarrow}$ production normalized to the SM rate as a function of $m_{A_{\downarrow}}$. The color code denotes the exclusion luminosity.

is due to our scan procedure) the rates are largely enhanced because of the large di-Higgs production cross sections. With increasing mass the rates decrease. The exclusion luminosities are high for exotic Higgs masses above $125 \mathrm{GeV}$ and below the top-pair threshold. Above the top-pair threshold the exclusion luminosities are much lower due to the exclusion limits in the top-pair final state. For masses below the SM-like Higgs mass, however, there are parameter points where the exclusion luminosities can exceed $100 \mathrm{fb}^{-1}$ and even $1 \mathrm{ab}^{-1}$ while still featuring large rates. The reason is that these points are not excluded from single-Higgs searches as light Higgs states with dominant decays into $b \bar{b}$ final states are difficult to probe. On the other hand this enhancement combined with the large di-Higgs production cross section implies huge $4 b$ finalstate rates, that may be tested at high luminosities, but with associated experimental difficulties. This is a nice example of the interplay between difficult single-Higgs searches and large exotic di-Higgs rates, where new physics may be found.

\section{BENCHMARKS AND PHENOMENOLOGY}

\section{A. Type 1 benchmarks}

We describe a representative set of benchmarks of the C2HDM T1 model and their associated (exotic) multiHiggs phenomenology. The input parameters, the derived third neutral Higgs boson mass, the $C P$-odd admixtures in terms of the squared mixing matrix elements $R_{i 3}^{2}$ and the exclusion luminosity $\mathcal{L}_{\text {excl }}$ are given in Table VI. We also give the NLO QCD gluon-fusion $h h$ production cross section at $\sqrt{s}=14 \mathrm{TeV}$ together with its $K$ factor, given by the ratio of the NLO cross section to the LO one. In Table VII we present the $4 b,(2 b)(2 \tau)$ and $(2 b)(2 \gamma)$ rates from Higgs pair production normalized to the rate expected in the SM from Higgs pairs relevant for the discussion of the various benchmark points. 
TABLE VI. T1: Rows 1-8: The input parameters of the benchmark points T1BP1-6. Rows 9-13: The derived third neutral Higgs boson mass, the $C P$-odd admixtures $R_{i 3}^{2}$ and the exclusion luminosity $\mathcal{L}_{\text {excl }}$. Rows 14-15: The NLO QCD gluon-fusion $h h$ production cross section at $\sqrt{s}=14 \mathrm{TeV}$ and the corresponding $K$ factor.

\begin{tabular}{|c|c|c|c|c|c|c|}
\hline & T1BP1 & T1BP2 & T1BP3 & T1BP4 & T1BP5 & T1BP6 \\
\hline$m_{H_{1}}[\mathrm{GeV}]$ & 125.09 & 125.09 & 125.09 & 119.73 & 125.09 & 62.67 \\
\hline$m_{\mathrm{H}_{2}}[\mathrm{GeV}]$ & 130.24 & 131.52 & 233.86 & 125.09 & 265.60 & 125.09 \\
\hline$m_{H^{ \pm}}[\mathrm{GeV}]$ & 169.99 & 282.75 & 164.87 & 185.41 & 307.47 & 164.35 \\
\hline $\operatorname{Re}\left(m_{12}^{2}\right)\left[\mathrm{GeV}^{2}\right]$ & 679 & 12376 & 11473 & 7522 & 11435 & 130 \\
\hline$\alpha_{1}$ & 1.300 & 1.249 & 1.268 & 1.276 & 1.246 & -0.145 \\
\hline$\alpha_{2}$ & -0.075 & -0.032 & 0.00262 & 1.494 & $7.125 \times 10^{-3}$ & -0.0536 \\
\hline$\alpha_{3}$ & 1.306 & 1.570 & -0.809 & -1.460 & -1.478 & -0.0650 \\
\hline $\tan \beta$ & 4.05 & 3.23 & 3.32 & 5.30 & 5.54 & 8.26 \\
\hline$m_{H_{3}}[\mathrm{GeV}]$ & 132.95 & 290.17 & 234.51 & 211.43 & 279.70 & 138.01 \\
\hline$R_{13}^{2}$ & $5.558 \times 10^{-3}$ & $1.027 \times 10^{-3}$ & $6.863 \times 10^{-6}$ & 0.994 & $5.077 \times 10^{-5}$ & $2.870 \times 10^{-3}$ \\
\hline$R_{23}^{2}$ & 0.926 & 0.999 & 0.523 & $5.819 \times 10^{-3}$ & 0.991 & $4.212 \times 10^{-3}$ \\
\hline$R_{33}^{2}$ & 0.068 & $1.217 \times 10^{-6}$ & 0.477 & $7.140 \times 10^{-5}$ & $8.611 \times 10^{-3}$ & 0.993 \\
\hline $\mathcal{L}_{\text {excl }}\left[\mathrm{fb}^{-1}\right]$ & 11500 & 1641 & 1365 & 2628 & 1082 & 2579 \\
\hline$\sigma_{h h}^{\mathrm{NLO}}[\mathrm{fb}]$ & 36.52 & 36.59 & 37.88 & 38.21 & 897.74 & 37.26 \\
\hline$K$-factor & 1.95 & 1.95 & 1.95 & 1.95 & 2.06 & 1.95 \\
\hline
\end{tabular}

TABLE VII. C2HDM T1, T1BP1-6: The rates $(x x)(y y)_{H_{i} H_{j}}$ into the di-Higgs states $\left[H_{i} H_{j}\right]$ normalized to the SM from NLO QCD di-Higgs production through gluon fusion at $\sqrt{s}=14 \mathrm{TeV}$ in the final state $(x x)(y y):\left[p p \rightarrow H_{i} H_{j} \rightarrow(x x)(y y)\right] /[p p \rightarrow$ $\left.H^{\mathrm{SM}} H^{\mathrm{SM}} \rightarrow(x x)(y y)\right]$.

\begin{tabular}{lllllll}
\hline \hline & T1BP1 & T1BP2 & T1BP3 & T1BP4 & T1BP5 & T1BP6 \\
\hline$(b \bar{b})(b \bar{b})_{H_{i} H_{j}}[\mathrm{fb}]$ & {$[h h]: 1.05$} & {$\left[h H_{\downarrow}\right]: 1.69$} & {$\left[h H_{\downarrow}\right]: 0.026$} & {$\left[h H_{\downarrow}\right]: 0.66$} & {$[h h]: 23.80$} & {$\left[H_{\downarrow} H_{\downarrow}\right]: 145$} \\
$(b \bar{b})(\tau \bar{\tau})_{H_{i} H_{j}}[\mathrm{fb}]$ & {$[h h]: 1.04$} & {$\left[h H_{\downarrow}\right]: 1.70$} & {$\left[h H_{\downarrow}\right]: 0.027$} & {$\left[h H_{\downarrow}\right]: 0.66$} & {$[h h]: 23.51$} & {$\left[H_{\downarrow} H_{\downarrow}\right]: 124$} \\
$(b \bar{b})(\gamma \gamma)_{H_{i} H_{j}}[\mathrm{fb}]$ & {$[h h]: 0.91$} & {$\left[h H_{\downarrow}\right]: 0.97$} & {$\left[h H_{\downarrow}\right]: 0.019$} & {$\left[h H_{\downarrow}\right]: 0.41$} & {$[h h]: 24.32$} & {$\left[H_{\downarrow} H_{\downarrow}\right]: 0.29$} \\
\hline \hline
\end{tabular}

(1) T1BP1-Highest_exclusion_lumi: The point with the highest exclusion luminosity in the complete sample.

The exclusion luminosity for this point is found to be $11.5 \mathrm{ab}^{-1}$, i.e., well above the LHC design luminosity even after the high-luminosity phase. All di-Higgs cross sections involving non-SM-like Higgs bosons have values below the SM reference point. Altogether this benchmark point behaves very SM-like as expected for such a high exclusion luminosity. The neutral Higgs mass spectrum is relatively degenerate with all masses in the vicinity of the SM-like Higgs boson at approximately $125 \mathrm{GeV}$. The SM-like Higgs pair production cross section for this point is SM-like: $b \bar{b} \gamma \gamma$ is about $10 \%$ below the SM expectation while $b \bar{b} \tau \tau$ and $b \bar{b} b \bar{b}$ are slightly enhanced by $5 \%$, cf. Table VII. The cases when an SM Higgs is accompanied by an additional exotic Higgs are around $30 \%$ of the SM expectation in $b \bar{b} \tau \tau$. With the latest improvements in hadronic tau tagging [160-162] such a signature might be phenomenologically accessible at the LHC with large luminosity.

(2) T1BP2-HighLMaxHsmHl: Among the points with exclusion luminosities $\geq 1 \mathrm{ab}^{-1}$ the point with the maximum cross section $g g \rightarrow h H_{\downarrow}$.

We have $\sigma\left(g g \rightarrow h H_{\downarrow}\right)=28.47 \mathrm{fb}$ at LO. As summarized in Table VII, for the final states involving $b$ 's, $\tau$ 's and $\gamma$ 's we find for this di-Higgs final state relative to the production of an SM Higgs pair with subsequent decay into the same final state

$$
\begin{aligned}
\sigma\left(g g \rightarrow h H_{\downarrow} \rightarrow 4 b\right) / \mathrm{SM} & =1.69, \\
\sigma\left(g g \rightarrow h H_{\downarrow} \rightarrow(2 b)(2 \tau)\right) / \mathrm{SM} & =1.70, \\
\sigma\left(g g \rightarrow h H_{\downarrow} \rightarrow(2 b)(2 \gamma)\right) / \mathrm{SM} & =0.97 .
\end{aligned}
$$

The neutral Higgs mass spectrum in this case is slightly split while the pair production of the SMlike Higgs bosons largely follows the SM paradigm. The mass of the lighter neutral state is rather close to the SM boson, which allows us to compare the rates 
with the SM itself. $H_{\downarrow}$ has a decay phenomenology that is SM-like. At $3 \mathrm{ab}^{-1}$ we can therefore expect around 26000 exotic $b \bar{b} \tau \tau$ events while the $b \bar{b} b \bar{b}$ rate is enhanced by $70 \%$ over the SM expectation.

(3) T1BP3-NonSMHsmHL: Among the points with exclusion luminosities $\geq 1 \mathrm{ab}^{-1}$ and $\Sigma_{H_{\downarrow}} \leq 0.5$ (i.e., dominantly non-SM-like decays for $H_{\downarrow}$ ) the point with the maximum cross section $g g \rightarrow h H_{\downarrow}$.

This point will have a highly non-SM decay phenomenology and cascade decays are an interesting avenue to look for such a scenario. All di-Higgs production cross sections involving non-SM-like Higgs bosons lie below $5 \mathrm{fb}$. While the point has interesting signatures for non-SM-like single-Higgs production, exotic di-Higgs production is not a very promising avenue. The cross sections are far below the SM value, as no resonance enhancement is possible as the Higgs mass values are too close. Additionally the branching ratios in SM-like Higgs states are very small, as decays into non-SM-like final states dominate. We have $\Sigma_{H_{\downarrow}}=0.180$, $\Sigma_{H_{\uparrow}}=0.149$. The SM-like Higgs pair production is consistent with the SM expectation within $10 \%$ for this point. Exotic production of $H_{\downarrow}$ together with the SM Higgs leads to around 220 exotic $(b \bar{b})(\tau \tau)$ events and around $1900(b \bar{b})(b \bar{b})$ at $3 \mathrm{ab}^{-1}$.

(4) T1BP4-MaxLEnhancedHsmHl: Among the points with $g g \rightarrow h H_{\downarrow} \geq 10 \mathrm{fb}$ the point with the maximum exclusion luminosity. The SM-like Higgs pair production modes fall again within $\sim 10 \%$ of the SM expectation. The mass of the lightest neutral Higgs boson of about $120 \mathrm{GeV}$ is again reasonably close to the SM-like Higgs to allow a direct comparison of expected rates, which are slightly smaller than the SM. Specifically, the exotic $b \bar{b} \tau \tau$ and $b \bar{b} b \bar{b}$ modes are $35 \%$ smaller than what we would expect for the SM mode with $m_{h} \simeq 125 \mathrm{GeV}$.

(5) T1BP5-EnhancedHsmHsm: Among the points with exclusion luminosities $\geq 1 \mathrm{ab}^{-1}$ the point with the maximum cross section $g g \rightarrow h h$.

The exotic Higgs bosons lie well above the SMlike Higgs state which opens the possibility of enhancing the SM-like di-Higgs production due to resonant enhancement of intermediate $H_{\downarrow / \uparrow}$ production. We find explicitly

$$
\begin{aligned}
\sigma(g g \rightarrow h h \rightarrow 4 b) / \mathrm{SM} & =23.80 \\
\sigma(g g \rightarrow h h & \rightarrow(2 b)(2 \tau)) / \mathrm{SM}=23.51 \\
\sigma(g g \rightarrow h h & \rightarrow(2 b)(2 \gamma)) / \mathrm{SM}=24.32
\end{aligned}
$$

(6) T1BP6-EnhancedHlHI: Among the points with exclusion luminosities $\geq 1 \mathrm{ab}^{-1}$ the point with the maximum cross section $g g \rightarrow H_{\downarrow} H_{\downarrow}$.
The exclusion luminosity is $2.58 \mathrm{ab}^{-1}$ and we have a light $H_{\downarrow}$ with a mass just above half the SMlike mass so that the branching ratios of the latter remain in accordance with the LHC data. The diHiggs production of $H_{\downarrow} H_{\downarrow}$ amounts to $1.249 \mathrm{pb}$ at LO QCD. Comparing to the SM expectation, we have

$$
\begin{aligned}
\sigma\left(g g \rightarrow H_{\downarrow} H_{\downarrow} \rightarrow 4 b\right) / \mathrm{SM} & =145, \\
\sigma\left(g g \rightarrow H_{\downarrow} H_{\downarrow} \rightarrow(2 b)(2 \tau)\right) / \mathrm{SM} & =124, \\
\sigma\left(g g \rightarrow H_{\downarrow} H_{\downarrow} \rightarrow(2 b)(2 \gamma)\right) / \mathrm{SM} & =0.29,
\end{aligned}
$$

due to the suppressed decay $H_{\downarrow} \rightarrow \gamma \gamma$. The light Higgs almost exclusively decays into $b$ pairs at a branching fraction of $85 \%$. This means that such a state is difficult to observe in single-Higgs production as trigger criteria typically remove such events from the busy hadronic LHC environment. There is a possibility to observe this state in its $\tau$ modes $(\simeq 8 \%)$. This point is a nice of example how the $4 b$ mode can be an important BSM discovery tool when SM mass correlations are relaxed.

We note that none of these points feature a strong firstorder electroweak phase transition. In general we observe that points with a strong first-order phase transition do not lead to enhanced rates in the four-particle final states: the cross section for SM-like di-Higgs production is close to the SM value while other di-Higgs production cross sections are smaller than the SM expectation.

In addition to these points which are all characterized by relatively light exotic states we include benchmarks with heavy neutral exotics. This is achieved by adding the additional requirement $m_{H_{\uparrow}} \geq 400 \mathrm{GeV}$ to the defining criteria of the benchmark points quoted above. We restrict ourselves to three benchmark points to highlight special features. They are called T1BP1 H, T1BP2 H and T1BP5_H in analogy to their lighter mass spectrum counterparts (T1BP1, T1BP2, and T1BP5). Detailed information on the benchmark points is summarized in Table VIII.

(1) T1BP1_H-Highest_exclusion_lumi: The exclusion luminosity for this point is found to be $2.46 \mathrm{ab}^{-1}$. All di-Higgs cross sections involving non-SM-like Higgs bosons have values below the SM reference as the Higgs pair production cross section falls steeply for heavy Higgs production. The additional resonant structures that are sourced in the SM-like Higgs pair production amount to an increase above the SM expectation by a factor of $\sim 5.8$ across the standard search channels $4 b$, $(2 b)(2 \tau),(2 b)(2 \gamma)$.

(2) T1BP2_H-HighLMaxHsmHl: We have $\sigma(g g \rightarrow$ $\left.h H_{\downarrow}\right)=2.34 \mathrm{fb}$ at $\mathrm{LO}$, which is rather large given the mass of the exotic Higgs. The decay 
TABLE VIII. T1 heavy spectrum. Rows 1-8: The input parameters of the benchmark points T1BP1_H, T1BP2_H and T1BP5_H. Rows 9-13: The derived third neutral Higgs boson mass, the $C P$-odd admixtures $R_{i 3}^{2}$ and the exclusion luminosity $\mathcal{L}_{\text {excl }}$. Rows 14-15: The NLO QCD gluon-fusion $h h$ production cross section at $\sqrt{s}=14 \mathrm{TeV}$ and the corresponding $K$ factor.

\begin{tabular}{lccc}
\hline \hline & T1BP1_H & T1BP2_H & T1BP5_H \\
\hline$m_{H_{1}}[\mathrm{GeV}]$ & 125.09 & 125.09 & 125.09 \\
$m_{H_{2}}[\mathrm{GeV}]$ & 407.30 & 364.98 & 397.67 \\
$m_{H^{ \pm}}[\mathrm{GeV}]$ & 410.24 & 436.72 & 428.96 \\
$\operatorname{Re}\left(m_{12}^{2}\right)\left[\mathrm{GeV}^{2}\right]$ & 17152 & 39827 & 17992 \\
$\alpha_{1}$ & 1.406 & 1.291 & 1.379 \\
$\alpha_{2}$ & $-5.946 \times 10^{-3}$ & $3.132 \times 10^{-3}$ & $5.435 \times 10^{-3}$ \\
$\alpha_{3}$ & 0.244 & -1.550 & -0.685 \\
$\tan \beta$ & 9.20 & 3.40 & 8.28 \\
$m_{H_{3}}[\mathrm{GeV}]$ & 425.13 & 401.33 & 403.92 \\
$R_{13}^{2}$ & $3.535 \times 10^{-5}$ & $9.809 \times 10^{-6}$ & $2.950 \times 10^{-5}$ \\
$R_{23}^{2}$ & 0.059 & 0.999 & 0.400 \\
$R_{33}^{2}$ & 0.941 & $4.330 \times 10^{-4}$ & 0.600 \\
$\mathcal{L}_{\text {excl }}^{2}\left[\mathrm{fb}{ }^{-1}\right]$ & 2461 & 1792 & 1590 \\
$\sigma_{h h}^{\mathrm{NLO}}[\mathrm{fb}]$ & 206.53 & 43.81 & 400.60 \\
$K$ factor & 1.98 & 1.98 & 1.99 \\
\hline \hline
\end{tabular}

phenomenology of the additional Higgs is completely dominated by decays into top final states. In this sense the single-Higgs production and exotic $h H_{\downarrow}$ production are fully correlated. The exclusion luminosity is $1.8 \mathrm{ab}^{-1}$ and results from the extrapolation of the $t \bar{t}$ resonance search. This point, although not relevant for di-Higgs analyses shows how single-Higgs measurements in the $t \bar{t}$ channel influence multi-Higgs final states. Such a benchmark could be adopted to further clarify the role of single Higgs measurements for exotic multi-Higgs final states.

(3) T1BP5_H-EnhancedHsmHsm: We have enhanced SM-like di-Higgs production due to resonant enhancement of intermediate $H_{\downarrow}$ and $H_{\uparrow}$ production. We find

$$
\begin{aligned}
\sigma(g g \rightarrow h h \rightarrow 4 b) / \mathrm{SM} & =11.22, \\
\sigma(g g \rightarrow h h & \rightarrow(2 b)(2 \tau)) / \mathrm{SM}=11.08, \\
\sigma(g g \rightarrow h h \rightarrow(2 b)(2 \gamma)) / \mathrm{SM} & =11.15 .
\end{aligned}
$$

This point hence gives access to SM-like Higgs pair production even for a heavy Higgs spectrum, and is an immediate sign of BSM physics as the di-Higgs cross section is enhanced.

\section{B. Type 2 benchmarks}

As already visible from Table IV, the C2HDM T2 model gives rise to less spectacular signatures than the C2HDM T1. We give two representative scenarios below. In general,
TABLE IX. C2HDM T2. Rows 1-8: The input parameters of the benchmark points T2BP1 and T2BP2. Rows 9-13: The derived third neutral Higgs boson mass, the $C P$-odd admixtures $R_{i 3}^{2}$ and the exclusion luminosity $\mathcal{L}_{\text {excl }}$. Rows $14-15$ : The NLO QCD gluon-fusion $h h$ production cross section at $\sqrt{s}=14 \mathrm{TeV}$ and the corresponding $K$ factor.

\begin{tabular}{lcc}
\hline \hline & T2BP1 & T2BP2 \\
\hline$m_{H_{1}}[\mathrm{GeV}]$ & 125.09 & 125.09 \\
$m_{H_{2}}[\mathrm{GeV}]$ & 858.09 & 814.56 \\
$m_{H^{ \pm}}[\mathrm{GeV}]$ & 835.85 & 894.84 \\
$\operatorname{Re}\left(m_{12}^{2}\right)\left[\mathrm{GeV}^{2}\right]$ & 252703 & 227697 \\
$\alpha_{1}$ & 1.141 & 1.042 \\
$\alpha_{2}$ & $-5.268 \times 10^{-4}$ & $6.184 \times 10^{-4}$ \\
$\alpha_{3}$ & 1.198 & -1.157 \\
$\tan \beta$ & 2.16 & 1.71 \\
$m_{H_{3}}[\mathrm{GeV}]$ & 858.65 & 814.94 \\
$R_{13}^{2}$ & $2.775 \times 10^{-7}$ & $3.824 \times 10^{-7}$ \\
$R_{23}^{2}$ & 0.867 & 0.832 \\
$R_{33}^{2}$ & 0.133 & 0.162 \\
$\mathcal{L}_{\text {excl }}^{2}$ & 2664 & 2016 \\
$\sigma_{h h}^{\text {NLO }}[\mathrm{fb}]$ & 37.82 & 38.02 \\
$K$ factor & 1.95 & 1.95 \\
\hline \hline
\end{tabular}

the spectrum is much heavier than for T1. There is no scenario where the SM-like Higgs boson is $\mathrm{H}_{2}$ : the SM Higgs is always the lightest state $H_{1}$. The input parameters for these points as well as further relevant information are summarized in Table IX.

(1) T2BP1-Highest_exclusion_lumi: The exclusion luminosity for this point is found to be $2.66 \mathrm{ab}^{-1}$. All di-Higgs cross sections involving non-SM-like Higgs bosons have values below the SM reference value. Altogether this benchmark point behaves very SM-like as expected for such a high exclusion luminosity where $t \bar{t}$ resonance searches become sensitive to this scenario.

(2) T2BP2-EnhancedHSMHSM: The di-Higgs production into an SM-like Higgs pair is enhanced so that we get

$$
\begin{aligned}
\sigma(g g \rightarrow h h \rightarrow 4 b) / \mathrm{SM} & =1.101, \\
\sigma(g g \rightarrow h h \rightarrow(2 b)(2 \tau)) / \mathrm{SM} & =1.088 \\
\sigma(g g \rightarrow h h \rightarrow(2 b)(2 \gamma)) / \mathrm{SM} & =1.037
\end{aligned}
$$

Despite a lower exclusion luminosity this point also behaves very SM-like and barely exceeds the rates into SM-like final states of T2BP1 which has a higher exclusion luminosity.

Overall, it will be difficult to probe the C2HDM T2, which features a heavy Higgs spectrum, in di-Higgs production. This is also partly due to the fact that enhanced SM-like Higgs pair production cross sections are already 
TABLE X. NMSSM. Rows 1-14: The input parameters of the benchmark points NMBP1-4. Rows 15-20: The derived Higgs boson masses. Row 21: The exclusion luminosity. Rows 22-23: The NLO QCD gluon-fusion $h h$ production cross section at $\sqrt{s}=14 \mathrm{TeV}$ and its corresponding $K$ factor.

\begin{tabular}{lcccc}
\hline \hline & NMBP1 & NMBP2 & NMBP3 & NMBP4 \\
\hline$M_{1}[\mathrm{GeV}]$ & 638 & 457 & 608 & 313 \\
$M_{2}[\mathrm{GeV}]$ & 1254 & 386 & 546 & 569 \\
$M_{3}[\mathrm{GeV}]$ & 4169 & 6345 & 6778 & 3485 \\
$A_{t}[\mathrm{GeV}]$ & 2456 & 5134 & 1092 & 532 \\
$A_{b}[\mathrm{GeV}]$ & -2213 & -2908 & -4015 & 2009 \\
$A_{\tau}[\mathrm{GeV}]$ & 1443 & -667 & 2370 & 354 \\
$M_{\tilde{Q}_{3}}[\mathrm{GeV}]$ & 1293 & 3175 & 2574 & 3581 \\
$M_{\tilde{L}_{3}}[\mathrm{GeV}]$ & 1147 & 1276 & 790 & 1188 \\
$\tan \beta$ & 1.96 & 1.87 & 1.68 & 1.49 \\
$\lambda$ & 0.55 & 0.50 & 0.60 & 0.54 \\
$\kappa$ & 0.43 & 0.47 & 0.33 & 0.27 \\
$A_{\lambda}[\mathrm{GeV}]$ & -55 & 33 & 425 & -416 \\
$A_{\kappa}[\mathrm{GeV}]$ & 373 & 358 & -672 & 667 \\
$\mu_{\text {eff }}[\mathrm{GeV}]$ & -293 & -299 & 321 & -327 \\
$m_{H_{1}}[\mathrm{GeV}]$ & 124.34 & 124.11 & 101.13 & 39.52 \\
$m_{H_{2}}[\mathrm{GeV}]$ & 335.27 & 409.70 & 125.88 & 125.64 \\
$m_{H_{3}}[\mathrm{GeV}]$ & 530.39 & 465.57 & 627.95 & 634.32 \\
$m_{A_{1}}[\mathrm{GeV}]$ & 487.34 & 406.66 & 608.57 & 580.42 \\
$m_{A_{2}}[\mathrm{GeV}]$ & 540.58 & 553.09 & 624.77 & 631.97 \\
$m_{H^{ \pm}}[\mathrm{GeV}]$ & 520.47 & 426.32 & 621.81 & 628.97 \\
$\mathcal{L}_{\mathrm{excl}}\left[\mathrm{fb}{ }^{-1}\right]$ & 1370 & 118 & 1192 & 101 \\
$\sigma_{h h}^{\mathrm{NLO}}[\mathrm{fb}]$ & 69.29 & 131.83 & 43.62 & 42.31 \\
$K$ factor & 1.97 & 1.97 & 1.96 & 1.96 \\
\hline \hline
\end{tabular}

excluded by the LHC limits on resonant heavy scalar production with decays into an SM Higgs boson pair.

\section{NMSSM Benchmarks}

Let us finally turn to the NMSSM. The criteria for selecting the benchmark points are as follows.

(1) NMBP1: The point with the largest $4 b$ rate from SM-like Higgs boson pair production with an exclusion luminosity above $1 \mathrm{ab}^{-1}$.

(2) NMBP2: The same as BP1 but with an exclusion luminosity beyond $100 \mathrm{fb}^{-1}$.

(3) NMBP 3 : The point with the largest $4 b$ rate from the production of an SM-like Higgs boson and the lighter of the $C P$-even non-SM-like Higgs bosons, $H_{\downarrow}$, with an exclusion luminosity above $1 \mathrm{ab}^{-1}$.

(4) NMBP4: The point with the largest $4 b$ rate from $H_{\downarrow} H_{\downarrow}$ production with an exclusion luminosity above $100 \mathrm{fb}^{-1}$.

We also provide benchmark points for di-Higgs final states involving a light pseudoscalar $A_{\downarrow}$ in the final state.

(1) NMBP 5: The point with the largest $4 b$ rate from $h A_{\downarrow}$ production with an exclusion luminosity above $1 \mathrm{ab}^{-1}$.

(2) NMBP6: The same as BP5 but with an exclusion luminosity beyond $100 \mathrm{fb}^{-1}$.

(3) NMBP7: The point with the largest $4 b$ rate from $A_{\downarrow} A_{\downarrow}$ production and an exclusion luminosity above $1 \mathrm{ab}^{-1}$. It turns out that NMBP7 is identical to NMBP5.

(4) NMBP8 : The same definition as for NMBP7 but with an exclusion luminosity beyond $100 \mathrm{fb}^{-1}$.

The input values, the derived Higgs boson masses, the exclusion luminosity and the NLO QCD cross section for $h h$ production with its $K$ factor for the various benchmark points are listed in Tables X and XII. Note, that we include benchmark points with an exclusion luminosity around $100 \mathrm{fb}^{-1}$ when the rates are much enhanced compared to the SM as in this case a luminosity of $100 \mathrm{fb}^{-1}$ might be enough to test this parameter point. As the rates for diHiggs production involving a heavy scalar or pseudoscalar are low we do not present benchmarks for these cases.

From Table XI we can read off that the $4 b$ rates from $h h$ production for an exclusion luminosity above $100 \mathrm{fb}^{-1}$ (NMBP2) can be almost a factor of about 4.8 , and thus it will be difficult to access this process at the lower luminosity. Assuming an exclusion luminosity above $1 \mathrm{ab}^{-1}$ (NMBP1) the enhancement compared to the SM rate is around 2.4. Again, larger enhancements in the final state with an SM-like Higgs boson pair are excluded by the limits provided from ATLAS and CMS $[143,144]$. In the $h H_{\downarrow}$ final state the enhancement factor is only 2.3 as the diHiggs production cross section

$$
\text { NMBP3: } \sigma\left(h H_{\downarrow}\right)=49.13 \mathrm{fb} \quad(K=1.92),
$$

is not much larger than in the SM. For $H_{\downarrow} H_{\downarrow}$ production we have, however,

TABLE XI. NMSSM, NMBP1-4: The rates $(x x)(y y)_{H_{i} H_{j}}$ into the di-Higgs states $\left[H_{i} H_{j}\right]$ normalized to the SM from NLO QCD di-Higgs production through gluon fusion at $\sqrt{s}=14 \mathrm{TeV}$ in the final state $(x x)(y y):[p p \rightarrow$ $\left.H_{i} H_{j} \rightarrow(x x)(y y)\right] /\left[p p \rightarrow H^{\mathrm{SM}} H^{\mathrm{SM}} \rightarrow(x x)(y y)\right]$.

\begin{tabular}{lcccc}
\hline \hline & NMBP1 & NMBP2 & NMBP3 & NMBP4 \\
\hline$(b \bar{b})(b \bar{b})_{H_{i} H_{j}}[\mathrm{fb}]$ & {$[h h]: 2.35$} & {$[h h]: 4.77$} & {$\left[h H_{\downarrow}\right]: 2.33$} & {$\left[H_{\downarrow} H_{\downarrow}\right]: 541.60$} \\
$(b \bar{b})(\tau \bar{\tau})_{H_{i} H_{j}}[\mathrm{fb}]$ & {$[h h]: 2.31$} & {$[h h]: 4.70$} & {$\left[h H_{\downarrow}\right]: 2.27$} & {$\left[H_{\downarrow} H_{\downarrow}\right]: 432.18$} \\
$(b \bar{b})(\gamma \gamma)_{H_{i} H_{j}}[\mathrm{fb}]$ & {$[h h]: 2.10$} & {$[h h]: 3.79$} & {$\left[h H_{\downarrow}\right]: 1.16$} & {$\left[H_{\downarrow} H_{\downarrow}\right]: 7.11$} \\
\hline \hline
\end{tabular}


TABLE XII. NMSSM. Rows 1-14: The input parameters of the benchmark points NMBP5 , 6, 8 (NMBP7=NMBP5). Rows 15-20: The derived Higgs boson masses. Row 21: The exclusion luminosity. Rows 22-23: The NLO QCD gluon-fusion $h h$ production cross section at $\sqrt{s}=14 \mathrm{TeV}$ and its corresponding $K$ factor.

\begin{tabular}{lccc}
\hline \hline & NMBP5 & NMBP6 & NMBP8 \\
\hline$M_{1}[\mathrm{GeV}]$ & 455 & 842 & 321 \\
$M_{2}[\mathrm{GeV}]$ & 1741 & 1510 & 749 \\
$M_{3}[\mathrm{GeV}]$ & 4179 & 1924 & 2060 \\
$A_{t}[\mathrm{GeV}]$ & -5923 & -4901 & 5449 \\
$A_{b}[\mathrm{GeV}]$ & -3994 & -3817 & 1232 \\
$A_{\tau}[\mathrm{GeV}]$ & -1773 & -226 & 2253 \\
$M_{\tilde{Q}_{3}}[\mathrm{GeV}]$ & 2391 & 3539 & 2344 \\
$M_{\tilde{L}_{3}}[\mathrm{GeV}]$ & 2117 & 1623 & 1163 \\
$\tan \beta$ & 2.17 & 1.46 & 1.98 \\
$\lambda$ & 0.53 & 0.55 & 0.49 \\
$\kappa$ & 0.44 & 0.42 & 0.46 \\
$A_{\lambda}[\mathrm{GeV}]$ & -177 & -4.78 & 60.12 \\
$A_{\kappa}[\mathrm{GeV}]$ & 47 & 6.32 & -0.81 \\
$\mu_{\mathrm{eff}}[\mathrm{GeV}]$ & -327 & -307 & 418 \\
$m_{H_{1}}[\mathrm{GeV}]$ & 125.62 & 125.59 & 125.07 \\
$m_{H_{2}}[\mathrm{GeV}]$ & 504.14 & 428.17 & 618.30 \\
$m_{H_{3}}[\mathrm{GeV}]$ & 612.42 & 465.10 & 767.13 \\
$m_{A_{1}}[\mathrm{GeV}]$ & 220.91 & 106.06 & 37.38 \\
$m_{A_{2}}[\mathrm{GeV}]$ & 602.07 & 444.01 & 629.92 \\
$m_{H^{ \pm}}[\mathrm{GeV}]$ & 597.20 & 432.53 & 620.36 \\
$\mathcal{L}_{\text {excl }}\left[\mathrm{fb}{ }^{-1}\right]$ & 1153 & 152 & 109 \\
$\sigma_{h h}^{\mathrm{NLO}}[\mathrm{fb}]$ & 53.42 & 97.53 & 54.54 \\
$K$ factor & 1.96 & 1.97 & 1.96 \\
\hline \hline
\end{tabular}

$$
\operatorname{NMBP} 4: \sigma\left(H_{\downarrow} H_{\downarrow}\right)=8.96 \mathrm{pb} \quad(K=2.30),
$$

leading to an enhancement factor of up to 540. The $H_{\downarrow}$ mass is much lower here than in the former case which induces dominant branching ratios into $b \bar{b}$. The large cross section is mainly due to the small $H_{\downarrow}$ mass. The resonant enhancement plays a minor role here. Both benchmark points are special in the sense that the SM-like Higgs boson is not the lightest but the second lightest $C P$-even Higgs boson in the spectrum. We hence have a light $C P$-even Higgs boson in these scenarios. The $H_{\downarrow}$ is very singlet like in both cases and decays with a branching ratio of about 0.9 into $b \bar{b}$.

From Table XIII we can read off that the $4 b,(2 b)(2 \tau)$ and $(2 b)(2 \gamma)$ final states from both $h A_{\downarrow}$ and $A_{\downarrow} A_{\downarrow}$ production can be enhanced above the SM rate even for an exclusion luminosity of $1 \mathrm{ab}^{-1}$. For the lower exclusion luminosity the enhancement can be huge, in particular in the four-fermion final state from $A_{\downarrow} A_{\downarrow}$ production (NMBP8). These enhancements are due to large di-Higgs production cross sections which at NLO QCD amount to

$$
\begin{gathered}
\operatorname{NMBP} 5: \sigma\left(h A_{\downarrow}\right)=56.04 \mathrm{fb} \quad(K=1.93), \\
\sigma\left(A_{\downarrow} A_{\downarrow}\right)=74.34 \mathrm{pb} \quad(K=1.94), \\
\text { NMBP6: } \sigma\left(h A_{\downarrow}\right)=988 \mathrm{fb} \quad(K=1.99), \\
\operatorname{NMBP8}: \sigma\left(A_{\downarrow} A_{\downarrow}\right)=34.35 \mathrm{pb} \quad(K=2.30) .
\end{gathered}
$$

The enhanced di-Higgs cross section values are on the one hand due to the light pseudoscalar masses and on the other hand due to resonant scalar production for $A_{\downarrow} A_{\downarrow}$ or pseudoscalar production for $h A_{\downarrow}$ production. As already noted in the discussion of Fig. 4 this is an example where new physics may lead to huge measurable effects in Higgs pair production while the single-Higgs process, here $A_{\downarrow}$ production in gluon fusion, is difficult to access. This is a prime example that demonstrates that, despite the very SMlike nature of the $125 \mathrm{GeV}$ Higgs boson, Higgs pair production can be far from being SM-like.

We finally remark that in all NMSSM scenarios the topsquark masses are quite large, of the order of $1 \mathrm{TeV}$ and larger.

\section{CONCLUSIONS}

Multi-Higgs final states are statistically limited at the LHC, but are key processes to gain a precise understanding of the mechanism of electroweak symmetry breaking. Phenomenologically, they are highly correlated with measurements in single-Higgs final states. The question of how much additional information can be gained from the investigation of multi-Higgs final states is therefore best addressed using concrete BSM extensions.

TABLE XIII. NMSSM, NMBP5 , 6,8 (NMBP7=NMBP5): The rates $(x x)(y y)_{H_{i} H_{j}}$ into the di-Higgs states $\left[H_{i} H_{j}\right]$ normalized to the SM from NLO QCD di-Higgs production through gluon fusion at $\sqrt{s}=14 \mathrm{TeV}$ in the final state $(x x)(y y):\left[p p \rightarrow H_{i} H_{j} \rightarrow(x x)(y y)\right] /\left[p p \rightarrow H^{\mathrm{SM}} H^{\mathrm{SM}} \rightarrow(x x)(y y)\right]$.

\begin{tabular}{lllll}
\hline \hline & \multicolumn{2}{c}{ NMBP5 } & NMBP6 & NMBP8 \\
\hline$(b \bar{b})(b \bar{b})_{H_{i} H_{j}}[\mathrm{fb}]$ & {$\left[h A_{\downarrow}\right]: 2.56$} & {$\left[A_{\downarrow} A_{\downarrow}\right]: 4.57$} & {$\left[h A_{\downarrow}\right]: 44.37$} & {$\left[A_{\downarrow} A_{\downarrow}\right]: 2127$} \\
$(b \bar{b})(\tau \bar{\tau})_{H_{i} H_{j}}[\mathrm{fb}]$ & {$\left[h A_{\downarrow}\right]: 2.68$} & {$\left[A_{\downarrow} A_{\downarrow}\right]: 5.07$} & {$\left[h A_{\downarrow}\right]: 43.22$} & {$\left[A_{\downarrow} A_{\downarrow}\right]: 1710$} \\
$(b \bar{b})(\gamma \gamma)_{H_{i} H_{j}}[\mathrm{fb}]$ & {$\left[h A_{\downarrow}\right]: 2.06$} & {$\left[A_{\downarrow} A_{\downarrow}\right]: 3.20$} & {$\left[h A_{\downarrow}\right]: 22.65$} & {$\left[A_{\downarrow} A_{\downarrow}\right]: 11.30$} \\
\hline \hline
\end{tabular}


Another particularly relevant question when considering di-Higgs final states is whether they could be a key discovery tool for BSM interactions. This could happen at the LHC in situations when single-Higgs analyses are simply not competitive due to, e.g., trigger thresholds that might be mitigated in more complex multi-Higgs final states. Large branching ratios of additional scalars into SMlike Higgs bosons serve as an additional avenue to observe resonantly enhanced SM-like Higgs production. In these scenarios, the kinematic correlations are often significantly modified compared to the SM.

In this work we have performed a comprehensive scan over the complex 2HDM and the NMSSM, with a particular emphasis on the expected di-Higgs phenomenology in these models, taking into account a variety of current constraints and future projections. We found that in particular in the C2HDM type 1 models, the di-Higgs phenomenology can significantly differ from the SM expectation. The differences range from new signatures in SM-like search channels for light Higgs bosons all the way to new resonant structures in di-Higgs final states for larger exotic Higgs masses. In particular, in final states involving light Higgs bosons the multifermion final states can be significantly enhanced compared to the SM case. This is also the case in the NMSSM where we can have light scalars or pseudoscalars in the spectrum. In the C2HDM type 2 models with their heavy Higgs spectra, the multi-Higgs final states play a less dominant role as new physics discoveries typically occur in $t \bar{t}$ resonance searches before (exotic) di-Higgs production becomes relevant.

We have distilled our scans into a representative number of benchmark points that not only reflect the phenomenological possibilities that present themselves in nonstandard Higgs sectors, but also point to a particular range of phenomenological situations.

First, the expected sensitivity of the $t \bar{t}$ resonance search is a crucial factor in deciding the relevance of di-Higgs searches. The multi-Higgs signal is typically driven by topmediated gluon fusion. Therefore, the decay to top final states is directly correlated with a large di-Higgs cross section for resonant production as well as enhancements in the decay to photons for nonresonant production.
Second, when exotic Higgs masses fall below the $t \bar{t}$ threshold, di-Higgs final states typically follow the SM decay rates with compressed neutral Higgs masses. This highlights the necessity to achieve a high mass resolution in the standard search channels $(2 b)(2 b),(2 b)(2 \tau),(2 b)(2 \gamma)$, even when SM-mass correlations are abandoned. While di-Higgs production would be enhanced in this instance, providing clear evidence of the presence of BSM interactions, their precise nature would remain elusive to some extent.

Alternatively, additional Higgs exotics can create multiple resonant features leading to a large enhancement of the total SM Higgs pair production rate. The extrapolated signal-strength constraints locate viable candidates for enhanced di-Higgs production in $b \bar{b}$ final states, which are difficult to access experimentally in single-Higgs production. Here di-Higgs production can play a significant role as a discovery tool for BSM interactions due to smaller backgrounds and better kinematical handles.

Third, relatively light Higgs bosons with significant branching ratios can lead to a strong enhancement in multifermion final states. Such signatures are already studied by the experimental collaborations. Our results indicate the importance of these analyses in the future.

While we have specifically focused on di-Higgs production, it is clear that these scenarios can have interesting non-SM signatures that can be exploited to observe or constrain a certain parameter point in a more targeted, yet parameter-point-dependent way. We will leave this for future work.

\section{ACKNOWLEDGMENTS}

We want to thank Jonas Wittbrodt for providing us with the C2HDM data sample. P. B. acknowledges the support by the DFG-funded Doctoral School Karlsruhe School of Elementary and Astroparticle Physics: Science and Technology (KSETA). S.D. is supported by the U.S. Department of Energy under Grant Contract No. DESC0012704. C. E. is supported by the IPPP Associateship scheme and by the UK Science and Technology Facilities Council (STFC) under Grant No. ST/P000746/1.
[1] G. Aad et al. (ATLAS Collaboration), Phys. Lett. B 716, 1 (2012).

[2] S. Chatrchyan et al. (CMS Collaboration), Phys. Lett. B 716, 30 (2012).

[3] J. M. Cornwall, D. N. Levin, and G. Tiktopoulos, Phys. Rev. Lett. 30, 1268 (1973); 31, 572(E) (1973).

[4] J. M. Cornwall, D. N. Levin, and G. Tiktopoulos, Phys. Rev. D 10, 1145 (1974); 11, 972(E) (1975).
[5] B. W. Lee, C. Quigg, and H. B. Thacker, Phys. Rev. Lett. 38, 883 (1977).

[6] B. W. Lee, C. Quigg, and H. B. Thacker, Phys. Rev. D 16, 1519 (1977).

[7] M. J. G. Veltman, Acta Phys. Pol. B 12, 437 (1981).

[8] A. D. Sakharov, Pis'ma Zh. Eksp. Teor. Fiz. 5, 32 (1967); Usp. Fiz. Nauk 161, 61 (1991). 
[9] B. Grzadkowski, M. Iskrzynski, M. Misiak, and J. Rosiek, J. High Energy Phys. 10 (2010) 085.

[10] D. de Florian et al. (LHC Higgs Cross Section Working Group), arXiv:1610.07922.

[11] S. Dawson, C. Englert, and T. Plehn, arXiv:1808 .01324 .

[12] J. R. Ellis, M. K. Gaillard, and D. V. Nanopoulos, Nucl. Phys. B106, 292 (1976).

[13] M. A. Shifman, A. I. Vainshtein, M. B. Voloshin, and V. I. Zakharov, Yad. Fiz. 30, 1368 (1979) [Sov. J. Nucl. Phys. 30, 711 (1979)].

[14] A. I. Vainshtein, V. I. Zakharov, and M. A. Shifman, Usp. Fiz. Nauk 131, 537 (1980) [Sov. Phys. Usp. 23, 429 (1980)].

[15] M. B. Voloshin, Yad. Fiz. 44, 738 (1986) [Sov. J. Nucl. Phys. 44, 478 (1986)].

[16] B. A. Kniehl and M. Spira, Z. Phys. C 69, 77 (1995).

[17] J. Baglio, F. Campanario, S. Glaus, M. Muhlleitner, M. Spira, and J. Streicher, arXiv:1811.05692.

[18] S. Borowka, N. Greiner, G. Heinrich, S. Jones, M. Kerner, J. Schlenk, U. Schubert, and T. Zirke, Phys. Rev. Lett. 117, 012001 (2016); 117, 079901 (2016).

[19] S. Borowka, N. Greiner, G. Heinrich, S. P. Jones, M. Kerner, J. Schlenk, and T. Zirke, J. High Energy Phys. 10 (2016) 107.

[20] G. Heinrich, S. P. Jones, M. Kerner, G. Luisoni, and E. Vryonidou, J. High Energy Phys. 08 (2017) 088.

[21] J. Davies, G. Mishima, M. Steinhauser, and D. Wellmann, J. High Energy Phys. 01 (2019) 176.

[22] R. Bonciani, G. Degrassi, P. P. Giardino, and R. Grber, Phys. Rev. Lett. 121, 162003 (2018).

[23] C.-Y. Chen, S. Dawson, and I. M. Lewis, Phys. Rev. D 91, 035015 (2015).

[24] V. Barger, T. Han, P. Langacker, B. McElrath, and P. Zerwas, Phys. Rev. D 67, 115001 (2003).

[25] J. Baglio, A. Djouadi, R. Grober, M. M. Muhlleitner, J. Quevillon, and M. Spira, J. High Energy Phys. 04 (2013) 151.

[26] R. S. Gupta, H. Rzehak, and J. D. Wells, Phys. Rev. D 88, 055024 (2013).

[27] S. Di Vita, C. Grojean, G. Panico, M. Riembau, and T. Vantalon, J. High Energy Phys. 09 (2017) 069.

[28] L. Di Luzio, R. Grober, and M. Spannowsky, Eur. Phys. J. C 77, 788 (2017).

[29] V. Khachatryan et al. (CMS Collaboration), Report No. CMS-PAS-FTR-16-002, 2017.

[30] A. Adhikary, S. Banerjee, R. K. Barman, B. Bhattacherjee, and S. Niyogi, J. High Energy Phys. 07 (2018) 116.

[31] D. Goncalves, T. Han, F. Kling, T. Plehn, and M. Takeuchi, Phys. Rev. D 97, 113004 (2018).

[32] S. Homiller and P. Meade, arXiv:1811.02572.

[33] A. Azatov, R. Contino, G. Panico, and M. Son, Phys. Rev. D 92, 035001 (2015).

[34] W. Yao, arXiv:1308.6302.

[35] A. J. Barr, M. J. Dolan, C. Englert, D. E. Ferreira de Lima, and M. Spannowsky, J. High Energy Phys. 02 (2015) 016.

[36] A. Papaefstathiou, Phys. Rev. D 91, 113016 (2015).

[37] X. Zhao, Q. Li, Z. Li, and Q.-S. Yan, Chin. Phys. C 41, 023105 (2017).
[38] R. Contino et al., CERN Yellow Report, 2017, pp. 255440, DOI: 10.23731/CYRM-2017-003.255.

[39] S. Banerjee, C. Englert, M. L. Mangano, M. Selvaggi, and M. Spannowsky, Eur. Phys. J. C 78, 322 (2018).

[40] R. Grober and M. Muhlleitner, J. High Energy Phys. 06 (2011) 020.

[41] M. J. Dolan, C. Englert, and M. Spannowsky, Phys. Rev. D 87, 055002 (2013).

[42] C.-Y. Chen, S. Dawson, and I. M. Lewis, Phys. Rev. D 90, 035016 (2014).

[43] R. Grober, M. Muhlleitner, and M. Spira, J. High Energy Phys. 06 (2016) 080.

[44] E. Vryonidou and C. Zhang, J. High Energy Phys. 08 (2018) 036.

[45] U. Ellwanger and M. Rodriguez-Vazquez, J. High Energy Phys. 11 (2017) 008.

[46] T. D. Lee, Phys. Rev. D 8, 1226 (1973); 8, 516 (1973).

[47] J. F. Gunion, H. E. Haber, G. L. Kane, and S. Dawson, Front. Phys. 80, 1 (2000).

[48] G. C. Branco, P. M. Ferreira, L. Lavoura, M. N. Rebelo, M. Sher, and J. P. Silva, Phys. Rep. 516, 1 (2012).

[49] I. F. Ginzburg, M. Krawczyk, and P. Osland, in Proceedings of the LCWS 2002 (2002), pp. 703-706.

[50] D. Fontes, J. C. Romao, and J. P. Silva, J. High Energy Phys. 12 (2014) 043.

[51] L. Lavoura and J. P. Silva, Phys. Rev. D 50, 4619 (1994).

[52] F. J. Botella and J. P. Silva, Phys. Rev. D 51, 3870 (1995).

[53] A. W. El Kaffas, P. Osland, and O. M. Ogreid, Nonlin. Phenom. Complex Syst. 10, 347 (2007).

[54] D. Fontes, M. Muhlleitner, J. C. Romao, R. Santos, J. P. Silva, and J. Wittbrodt, J. High Energy Phys. 02 (2018) 073.

[55] U. Ellwanger, C. Hugonie, and A. M. Teixeira, Phys. Rep. 496, 1 (2010).

[56] M. Maniatis, Int. J. Mod. Phys. A 25, 3505 (2010).

[57] H. E. Haber and O. Stl, Eur. Phys. J. C 75, 491 (2015); 76, 312 (2016).

[58] J. Baglio, O. Eberhardt, U. Nierste, and M. Wiebusch, Phys. Rev. D 90, 015008 (2014).

[59] G. Aad et al. (ATLAS and CMS Collaborations), Phys. Rev. Lett. 114, 191803 (2015).

[60] H. E. Haber and H. E. Logan, Phys. Rev. D 62, 015011 (2000).

[61] O. Deschamps, S. Descotes-Genon, S. Monteil, V. Niess, S. T'Jampens, and V. Tisserand, Phys. Rev. D 82, 073012 (2010).

[62] F. Mahmoudi and O. Stal, Phys. Rev. D 81, 035016 (2010).

[63] T. Hermann, M. Misiak, and M. Steinhauser, J. High Energy Phys. 11 (2012) 036.

[64] M. Misiak et al., Phys. Rev. Lett. 114, 221801 (2015).

[65] M. Misiak and M. Steinhauser, Eur. Phys. J. C 77, 201 (2017).

[66] S. Dawson and M. Sullivan, Phys. Rev. D 97, 015022 (2018).

[67] M. Baak, J. Cth, J. Haller, A. Hoecker, R. Kogler, K. Mnig, M. Schott, and J. Stelzer (Gfitter Group), Eur. Phys. J. C 74, 3046 (2014).

[68] M. Tanabashi et al. (Particle Data Group), Phys. Rev. D 98, 030001 (2018). 
[69] A. Denner et al., Report No. LHCHXSWG-INT-2015-006, 2015.

[70] R. Coimbra, M. O. P. Sampaio, and R. Santos, Eur. Phys. J. C 73, 2428 (2013).

[71] P. M. Ferreira, R. Guedes, M. O. P. Sampaio, and R. Santos, J. High Energy Phys. 12 (2014) 067.

[72] I. P. Ivanov and J. P. Silva, Phys. Rev. D 92, 055017 (2015).

[73] P. Bechtle, O. Brein, S. Heinemeyer, G. Weiglein, and K. E. Williams, Comput. Phys. Commun. 181, 138 (2010).

[74] P. Bechtle, O. Brein, S. Heinemeyer, G. Weiglein, and K. E. Williams, Comput. Phys. Commun. 182, 2605 (2011).

[75] P. Bechtle, O. Brein, S. Heinemeyer, O. Stl, T. Stefaniak, G. Weiglein, and K. E. Williams, Eur. Phys. J. C 74, 2693 (2014).

[76] P. Bechtle, S. Heinemeyer, O. Stl, T. Stefaniak, and G. Weiglein, Eur. Phys. J. C 74, 2711 (2014).

[77] A. Djouadi, J. Kalinowski, and M. Spira, Comput. Phys. Commun. 108, 56 (1998).

[78] A. Djouadi, J. Kalinowski, M. Muehlleitner, and M. Spira, arXiv:1801.09506.

[79] M. Krause, M. Muhlleitner, R. Santos, and H. Ziesche, Phys. Rev. D 95, 075019 (2017).

[80] M. Muhlleitner, M. O. P. Sampaio, R. Santos, and J. Wittbrodt, J. High Energy Phys. 08 (2017) 132.

[81] S. Inoue, M. J. Ramsey-Musolf, and Y. Zhang, Phys. Rev. D 89, 115023 (2014).

[82] V. Andreev et al. (ACME Collaboration), Nature (London) 562, 355 (2018).

[83] M. Quiros, Helv. Phys. Acta 67, 451 (1994).

[84] G. D. Moore, Phys. Rev. D 59, 014503 (1998).

[85] P. Basler and M. Muhlleitner, Comput. Phys. Commun. 237, 62 (2019).

[86] S. F. King, M. Mühlleitner, R. Nevzorov, and K. Walz, Phys. Rev. D 90, 095014 (2014).

[87] R. Costa, M. Muhlleitner, M. O. P. Sampaio, and R. Santos, J. High Energy Phys. 06 (2016) 034.

[88] D. Azevedo, P. Ferreira, M. Margarete Mhlleitner, R. Santos, and J. Wittbrodt, arXiv:1808.00755.

[89] P. Z. Skands et al., J. High Energy Phys. 07 (2004) 036.

[90] B. C. Allanach et al., Comput. Phys. Commun. 180, 8 (2009).

[91] U. Ellwanger, J. F. Gunion, and C. Hugonie, J. High Energy Phys. 02 (2005) 066.

[92] U. Ellwanger and C. Hugonie, Comput. Phys. Commun. 175, 290 (2006).

[93] U. Ellwanger and C. Hugonie, Comput. Phys. Commun. 177, 399 (2007).

[94] D. Das, U. Ellwanger, and A. M. Teixeira, Comput. Phys. Commun. 183, 774 (2012).

[95] M. Mühlleitner, A. Djouadi, and Y. Mambrini, Comput. Phys. Commun. 168, 46 (2005).

[96] G. Belanger, F. Boudjema, C. Hugonie, A. Pukhov, and A. Semenov, J. Cosmol. Astropart. Phys. 09 (2005) 001.

[97] G. Aad et al. (ATLAS and CMS Collaborations), J. High Energy Phys. 08 (2016) 045.

[98] R. V. Harlander, S. Liebler, and H. Mantler, Comput. Phys. Commun. 184, 1605 (2013).
[99] R. V. Harlander, S. Liebler, and H. Mantler, Comput. Phys. Commun. 212, 239 (2017).

[100] M. Spira, A. Djouadi, D. Graudenz, and P. M. Zerwas, Nucl. Phys. B453, 17 (1995).

[101] R. V. Harlander and W. B. Kilgore, Phys. Rev. Lett. 88, 201801 (2002).

[102] C. Anastasiou and K. Melnikov, Nucl. Phys. B646, 220 (2002).

[103] R. V. Harlander and W. B. Kilgore, J. High Energy Phys. 10 (2002) 017.

[104] C. Anastasiou and K. Melnikov, Phys. Rev. D 67, 037501 (2003).

[105] V. Ravindran, J. Smith, and W. L. van Neerven, Nucl. Phys. B665, 325 (2003).

[106] C. Anastasiou, C. Duhr, F. Dulat, E. Furlan, T. Gehrmann, F. Herzog, and B. Mistlberger, J. High Energy Phys. 03 (2015) 091.

[107] C. Anastasiou, C. Duhr, F. Dulat, E. Furlan, F. Herzog, and B. Mistlberger, J. High Energy Phys. 08 (2015) 051.

[108] C. Anastasiou, C. Duhr, F. Dulat, E. Furlan, T. Gehrmann, F. Herzog, A. Lazopoulos, and B. Mistlberger, J. High Energy Phys. 05 (2016) 058.

[109] B. Mistlberger, J. High Energy Phys. 05 (2018) 028.

[110] M. Bonvini, A. S. Papanastasiou, and F. J. Tackmann, J. High Energy Phys. 11 (2015) 196.

[111] M. Bonvini, A. S. Papanastasiou, and F. J. Tackmann, J. High Energy Phys. 10 (2016) 053.

[112] S. Forte, D. Napoletano, and M. Ubiali, Phys. Lett. B 751, 331 (2015).

[113] S. Forte, D. Napoletano, and M. Ubiali, Phys. Lett. B 763, 190 (2016).

[114] R. Harlander, M. Kramer, and M. Schumacher, arXiv: 1112.3478.

[115] R. V. Harlander and W. B. Kilgore, Phys. Rev. D 68, 013001 (2003).

[116] S. Dittmaier, M. Krmer, and M. Spira, Phys. Rev. D 70, 074010 (2004).

[117] S. Dawson, C. B. Jackson, L. Reina, and D. Wackeroth, Phys. Rev. D 69, 074027 (2004).

[118] M. Wiesemann, R. Frederix, S. Frixione, V. Hirschi, F. Maltoni, and P. Torrielli, J. High Energy Phys. 02 (2015) 132.

[119] J. Baglio, R. Grober, M. Muhlleitner, D. T. Nhung, H. Rzehak, M. Spira, J. Streicher, and K. Walz, Comput. Phys. Commun. 185, 3372 (2014).

[120] G. Aad et al. (ATLAS Collaboration), J. High Energy Phys. 10 (2015) 054.

[121] M. Aaboud et al. (ATLAS Collaboration), Phys. Rev. D 94, 052009 (2016).

[122] G. Aad et al. (ATLAS Collaboration), Eur. Phys. J. C 75, 208 (2015).

[123] P. A. R. Ade et al. (Planck Collaboration), Astron. Astrophys. 571, A16 (2014).

[124] D. S. Akerib et al. (LUX Collaboration), Phys. Rev. Lett. 118, 021303 (2017).

[125] E. Aprile et al. (XENON Collaboration), Phys. Rev. Lett. 121, 111302 (2018).

[126] M. Ackermann et al. (Fermi-LAT Collaboration), Phys. Rev. Lett. 115, 231301 (2015). 
[127] G. Aad et al. (ATLAS Collaboration), Phys. Rev. D 92, 012006 (2015).

[128] S. Dittmaier et al. (LHC Higgs Cross Section Working Group), arXiv:1101.0593.

[129] M. Aaboud et al. (ATLAS Collaboration), J. High Energy Phys. 12 (2017) 024.

[130] A. M. Sirunyan et al. (CMS Collaboration), Phys. Lett. B 780, 501 (2018).

[131] V. Khachatryan et al. (CMS Collaboration), Phys. Rev. D 92, 032008 (2015).

[132] A. M. Sirunyan et al. (CMS Collaboration), Phys. Lett. B 779, 283 (2018).

[133] M. Aaboud et al. (ATLAS Collaboration), Phys. Lett. B 775, 105 (2017).

[134] M. Aaboud et al. (ATLAS Collaboration), J. High Energy Phys. 01 (2018) 055.

[135] M. Aaboud et al. (ATLAS Collaboration), Eur. Phys. J. C 78, 24 (2018).

[136] M. Aaboud et al. (ATLAS Collaboration), Eur. Phys. J. C 78, 293 (2018).

[137] M. Aaboud et al. (ATLAS Collaboration), Eur. Phys. J. C 78, 565 (2018).

[138] M. Aaboud et al. (ATLAS Collaboration), Phys. Rev. Lett. 119, 191803 (2017).

[139] A. Agostini, G. Degrassi, R. Grober, and P. Slavich, J. High Energy Phys. 04 (2016) 106.

[140] R. Grober, M. Muhlleitner, and M. Spira, Nucl. Phys. B925, 1 (2017).

[141] S. Dulat, T.-J. Hou, J. Gao, M. Guzzi, J. Huston, P. Nadolsky, J. Pumplin, C. Schmidt, D. Stump, and C. P. Yuan, Phys. Rev. D 93, 033006 (2016).

[142] M. Aaboud et al. (ATLAS Collaboration), J. High Energy Phys. 01 (2019) 030.

[143] (CMS Collaboration), Report No. CMS-PAS-HIG-17-009, 2017, http://cds.cern.ch/record/2292044.

[144] A. M. Sirunyan et al. (CMS Collaboration), Phys. Lett. B 781, 244 (2018).
[145] (CMS Collaboration), Report No. CMS-PAS-B2G-17006, 2017, http://cds.cern.ch/record/2296716.

[146] A. M. Sirunyan et al. (CMS Collaboration), Phys. Lett. B 778, 101 (2018).

[147] M. Aaboud et al. (ATLAS Collaboration), Phys. Rev. Lett. 121, 191801 (2018).

[148] (CMS Collaboration), Report No. CMS-PAS-B2G-17006, 2017.

[149] U. Baur, T. Plehn, and D. L. Rainwater, Phys. Rev. D 69, 053004 (2004).

[150] U. Baur, T. Plehn, and D. L. Rainwater, Phys. Rev. D 68, 033001 (2003).

[151] M. J. Dolan, C. Englert, and M. Spannowsky, J. High Energy Phys. 10 (2012) 112.

[152] A. J. Barr, M. J. Dolan, C. Englert, and M. Spannowsky, Phys. Lett. B 728, 308 (2014).

[153] D. E. Ferreira de Lima, A. Papaefstathiou, and M. Spannowsky, J. High Energy Phys. 08 (2014) 030.

[154] D. Wardrope, E. Jansen, N. Konstantinidis, B. Cooper, R. Falla, and N. Norjoharuddeen, Eur. Phys. J. C 75, 219 (2015).

[155] U. Baur, T. Plehn, and D. L. Rainwater, Phys. Rev. Lett. 89, 151801 (2002).

[156] U. Baur, T. Plehn, and D. L. Rainwater, Phys. Rev. D 67, 033003 (2003).

[157] A. Papaefstathiou, L. L. Yang, and J. Zurita, Phys. Rev. D 87, 011301 (2013).

[158] A. M. Sirunyan et al. (CMS Collaboration), arXiv:1811 .08459 .

[159] A. M. Sirunyan et al. (CMS Collaboration), J. High Energy Phys. 09 (2018) 007.

[160] L. Cadamuro (CMS Collaboration), Proc. Sci. EPSHEP2015 (2015) 226.

[161] V. Khachatryan et al. (CMS Collaboration), https://cds .cern.ch/record/2018400.

[162] L. Mastrolorenzo, Nucl. Part. Phys. Proc. 273-275, 2518 (2016). 